\title{
EQUIVARIANT CONCORDANCE OF INVARIANT KNOTS
}

BY

\author{
NEAL W. STOLTZFUS
}

\begin{abstract}
The classification of equivariant concordance classes of high-dimensional codimension two knots invariant under a cyclic action, $T$, of order $m$ has previously been reported on by Cappell and Shaneson [CS2]. They give an algebraic solution in terms of their algebraic $K$-theoretic $\Gamma$-groups. This work gives an alternative description by generalizing the well-known Seifert linking forms of knot theory to the equivariant case. This allows explicit algorithmic computations by means of the procedures and invariants of algebraic number theory (see the subsequent work [St], particularly Theorem 6.13). Following Levine [L3], we define bilinear forms on the middle-dimensional homology of an equivariant Seifert surface $B_{i}(x, y)=$ $L\left(x, i_{+}\left(T_{*}^{i} y\right)\right)$, for $i=1, \ldots, m$. Our first result (2.5) is that an invariant knot is equivariantly concordant to an invariant trivial knot if and only if there is a subspace of half the rank on which the $B_{i}$ vanish simultaneously. We then introduce the concepts of equivariant isometric structure and algebraic concordance which mirror the preceding geometric ideas. The resulting equivalence classes form a group under direct sum which has infinitely many elements of each of the possible orders (two, four and infinite), at least for odd periods. The central computation (3.4) gives an isomorphism of the equivariant concordance group with the subgroup of the algebraic knot concordance group whose Alexander polynomial, $\Delta$, satisfies the classical relation $\left|\Pi_{i=1}^{m} \Delta\left(\lambda^{i}\right)\right|=1$, where $\lambda$ is a primitive $m$ th root of unity. This condition assures that the $m$-fold cover of the knot complement is also a homology circle, permitting the geometric realization of each equivariant isometric structure. Finally, we make an explicit computation of the Browder-Livesay desuspension invariant for knots invariant under an involution and also elucidate the connection of our methods with the results of [CS2] by explicitly describing a homomorphism from the group of equivariant isometric structures to the appropriate $\Gamma$-group.
\end{abstract}

Introduction. The initial impetus and inspirational origins for this paper are found in the work of Santiago Lopez de Medrano on codimension two knots invariant under involutions [LdM1]-[LdM3] and in that of William Browder on homotopy lens spaces, particularly in the philosophical directions indicated in the last paragraphs of the introduction to his paper in the Proceedings of the 1969 Georgia Conference on the Topology of Manifolds [Br3]. Also very influential in the development were the works of $\mathbf{H}$. Seifert [Sei], $\mathbf{R}$.

Received by the editors August 10, 1976.

AMS (MOS) subject classifications (1970). Primary 57C45, 57D40, $57 D 85$.

Key words and phrases. Knots, linking numbers, concordance, cyclic action, codimension two embeddings, lens spaces, isometric structures. 
H. Fox [F1]-[F3], M. Kervaire [K1], [K2], J. Levine [L1]-[L5] and J. W. Milnor [M3], [M4] in their studies on codimension two knotting phenomena. The problem of equivariant knot concordance also inspired the work of $S$. Cappell and J. Shaneson on abstract surgery in codimension two [CS2]. Their paper gave an algebraic computation of the equivariant knot concordance group using their idea of $\Gamma$-group and proved many results on the existence and enumeration of codimension two invariant subspheres. Our work gives an alternative algebraic solution to this problem which is very amenable to explicit algorithmic computation using known techniques of algebraic number theory [St].

The setting for this paper is the category of triples $\left(J^{2 n+1}, K ; T\right)$ where $(J, K)$ is a codimension two (locally flat) knot and $T$ is a free cyclic action of order $m$ leaving $K$ invariant. We may make this definition in any of the three topological categories of manifolds, DIFF, PL or TOP. Two triples with the same ambient space are called equivariantly concordant if there is an $h$ cobordism connecting them in $J \times I$ invariant under $T \times I d$. A triple is said to be a trivial invariant knot if the $\operatorname{knot}(J, K)$ is a trivial knot (with no condition on the action). We extend ideas of Jerome Levine to classify these triples up to equivariant concordance, the appropriate generalization of knot concordance (often called "knot cobordism") to the equivariant setting.

In the first two chapters, we develop an a priori obstruction theory using linking numbers to characterize those knots which are equivariantly concordant to a trivial invariant knot. Let $V^{2 n}$ be an equivariant Seifert manifold for $(J, K ; T)$ and $i_{+}$a small push in the positive normal direction. Define bilinear forms on $H_{n}\left(V^{2 n} ; Z\right)$ by

$$
B_{i}(x, y)=L\left(x, i_{+*}\left(T_{*}^{i} y\right)\right) \quad(i=0, \ldots, m-1)
$$

where $L$ is the linking bilinear form.

THEOREM 2.5. $(n>2)(J, K ; T)$ is equivariantly concordant to a trivial invariant knot iff there is a subspace $H$ in $H_{n}(V)$ of one-half the rank on which the $B_{i}$ vanish simultaneously $(i=0, \ldots, m-1)$.

This theorem is proven using techniques of equivariant surgery on the framed complement of $K$ in $J$. It is interesting to note that no distinction is made according to the parity of the order of the action.

In Chapter III the concept of equivariant isometric structure is introduced, generalizing an idea of Kervaire [K2] applied to ordinary knot concordance theory. Equivalence classes of these gadgets under the relation of algebraic concordance are shown to form a group $E^{e}(m ; Z)\left(\varepsilon=(-1)^{n}\right)$ with respect to the direct sum operation. Algebraic concordance faithfully mimics the geometry according to 
TheOREM 3.4. Let $(J, K ; T)$ and $B_{i}$ be as in Theorem 2.5. There exists a well-defined element $\alpha(J, K ; T)$ in $E^{\varepsilon}(m ; Z)$ depending only on the equivariant concordance class of $(J, K ; T)$ such that $\alpha(J, K ; T)=0$ iff there is a subspace $H$ in $H_{n}(V)$ of one-half the rank on which the $B_{i}$ vanish simultaneously.

The study of the group $E^{e}(m ; Z)$ is the focus of the remainder of Chapter III.

TheOREM 3.7. $E^{\varepsilon}(m ; Z)$ (and also the groups $\Gamma_{2 n}(Z \rightarrow Z / m Z$ ) of Cappell and Shaneson [CS2]) is contained in an infinite direct sum of infinitely many copies each of $Z, Z / 2 Z$ and $Z / 4 Z$.

An infinite number of elements of each order exists in $E^{e}(m ; Z)$ except possibly for elements of order 4 if $m$ is even.

An isometry is an endomorphism $t$ of a free $Z$-module $M$ with an $\varepsilon$-symmetric unimodular bilinear integer valued form $Q$ satisfying

$$
Q(t(x), y)+Q(x, t(y))=Q(x, y) .
$$

The Alexander polynomial of an isometry, defined by $(-\varepsilon)^{h} X^{2 h} \Delta\left(1-X^{-1}\right)$ $=f(X)$ where $f$ is the characteristic polynomial of $t$ of degree $2 h$, plays an important role. In particular, we have the relation

$$
\left|\prod_{i=1}^{m} \Delta\left(\zeta^{i}\right)\right|=1
$$

for $\zeta$ a primitive $m$ th root of unity. This is a necessary and sufficient condition on the isometry $t$ to define an equivariant isometric structure and is equivalent, in terms of the $\Gamma$-groups, to the unimodularity of the intersection form over $Z[Z / m Z]$. Using this one may demonstrate, using an inclusiontransfer sequence, that there are an infinite number of invariant knots not equivariantly concordant to $m$ times a knot and that ordinary knot concordance is infinitely generated over equivariant knot concordance under the transfer.

It is interesting to note that $(*)$ reflects an "Adams oepration" $\psi^{m}$ in ordinary (nonequivariant) knot concordance theory induced by the usual definition on bilinear forms. It is also curious that $\hat{\Delta}$ in the relation $\hat{\Delta}\left(X^{m}\right)=$ $\Pi_{i=1}^{m} \Delta\left(\zeta^{i} X\right)$ (used to prove (*)) is obtained by the same formal process, on the roots of $\Delta$, with symmetric functions as used in the definition of Adams operations in $K$-theory. Note that $\hat{\Delta}$ is not always a knot polynomial (see Chapter III) even if $\Delta$ is and, hence, $\psi^{m}$ is not always defined within knot concordance theory.

We now turn to the associated geometric operation in knot theory, that of "Frobenius", or taking the $m$-fold cover of a knot complement. (This action is also visible in Chapter III in the discussion preceding Theorem 3.7 where a certain isometry is shown to be the $m$ th power of another isometry. These 
isometries correspond geometrically to the action of the translation on the homology of the appropriate infinite cyclic cover.) (*) is the necessary and sufficient condition for the $m$-fold cover of a knot complement to be a homology circle and is the crucial observation in the proof of the following realization theorem for the algebraic construction of Chapter III.

TheOREM 4.1 $(n>2)$. Given $\left(K^{2 n-1}, T_{0}\right)$ a free PL action of order $m$ on a homotopy sphere $K$ and $\beta$ in $E^{\varepsilon}(m, Z)$, there exists an action $(J, T)$ on a homotopy sphere $J$ and an equivariant (locally flat) embedding $\left(K, T_{0}\right) \hookrightarrow(J, T)$ such that $\alpha(J, K ; T)=\beta$.

A complete discussion is also given in the smooth case. An attempt is made to determine the invariants for the PL classification of $(J, T)$ according to Browder, Petrie and Wall [BPW] in terms of those of $\left(K, T_{0}\right)$ and invariants of the knot and the normal bundle which succeeds for the torsion invariant, but the multisignature is only computed theoretically (in Chapter V).

In Chapter V, we connect our results with those of Santiago Lopez de Medrano [LdM1] [LdM3] for the case $m=2$ and the work on abstract codimension two surgery of S. Cappell and J. Shaneson [CS1], [CS2] and M. Kato and Y. Matsumoto [KaMa], [Ma]. First we make the interesting computation:

Proposition 5.1. Let $\left(\mathrm{J}^{4 k+3}, \mathrm{~K} ; \mathrm{T}\right)$ be a knot invariant under an involution. Then the Browder-Livesay desuspension invariant $=$ signature of $B_{1}(x, y)(=$ $\left.L\left(x, T_{*} y\right)\right)=$ signature of the orbit knot complement.

In the second section of Chapter $\mathrm{V}$, we relate our group to the algebraic $K$-theoretic group of Cappell and Shaneson in [CS2]:

Proposition 5.4.

$$
(n>2) \quad E^{\varepsilon}(m ; Z)=\Gamma_{2 n}^{h}\left(\begin{array}{ccc}
Z[Z] & \rightarrow & Z[Z] \\
\downarrow & & \downarrow \\
Z[Z] & \rightarrow & Z[Z / m Z]
\end{array}\right) .
$$

Then, using a generalization of a result of Wall [W3] and Trotter [T], we compute the intersection form in $\Gamma_{2 n}(Z \rightarrow Z / m Z)$ of the normal cobordism associated to an invariant knot. Because of the nature of $Z[Z]$ this determines an element in $\Gamma_{2 n}^{h}$ except for the Kervaire Arf invariant when $n$ is odd.

Finally we mention a further application of our methods to the problem of doing surgery on the complement up to homotopy equivalence (instead of homology equivalence) and obtain an algebraic obstruction monoid. The latter may be easily generalized to other cases, although no nontrivial com- 
putations are known. Finally, we note that the methods in this paper also apply to the setting of semifree actions on homotopy spheres with codimension sphere as fixed point set (see also [CS2]), as all considerations were made on the complements where the action is free.

One of the most intriguing aspects of this investigation was the convergence of many ideas current in topology: Galois theory, covering spaces, algebraic number theory, the Froebenius automorphism and Adams operations all impinging on the concordance theory of knots. The further understanding of these concepts and their interrelationships in knot theory should be beneficial in furthering our knowledge of codimension two phenomena.

The author gratefully acknowledges the support and inspiration of William Browder while writing this paper and wishes to thank Sylvain Cappell, Wu Chung Hsiang, William Pardon, Michael Freedman and Leonide Goldstein for illuminating conversations. We also acknowledge the contribution of C. C. Liang, Eva Bayer and friend. This work was partially supported by the National Science Foundation and contains results of a Princeton University thesis submitted in June, 1973.

\section{CHAPTER I: LINKING NUMBERS REVISITED}

In this chapter we will organize the necessary facts concerning "linking" in odd dimensional manifolds. The results of this chapter are either of the folklore genre or widely scattered in disjoint portions of the mathematical literature. We will phrase the results in terms of the more geometrical concepts of homology and intersection theory in manifolds rather than the Poincaré dual setting of cohomology and cup products as the former is more suited to our later constructions. We will assume the standard treatment of intersection theory for cycles in manifolds as given oroginally by Lefschetz [Lef] or in a modern form by Dold [D]. Finally, all coefficients for homology will be the integers, denoted $Z$, unless otherwise explicitly stated.

Linking phenomena occur in the following special situations: two disjoint cycles $x^{p}$ and $y^{q}$ (of dimension $p$ and $q$, respectively) in a manifold $M^{n}$ of dimension $n=p+q+1$ may link if

(a) $x$ is a torsion cycle so that the linking number $L(x, y)$ is in $Q / Z$, or

(b) $x$ is a boundary, $H_{p+1}(M)=0$, then $L(x, y)$ is an integer.

The second situation is the focus of this chapter.

We will henceforth assume the following specialized situation: $x^{p}$ and $y^{q}$ (of dimension $p$ and $q$, respectively) are both null-homologous disjoint (i.e. with disjoint carriers) singular cycles in an oriented manifold $M^{n}$ of dimension $n=p+q+1$ with $H_{p+1}(M)=0$.

Definition 1.1. The linking number of $x$ and $y$, denoted $L(x, y)$, is the integer defined as follows: since $x$ is a null-homologous cycle there exists a chain $z$ with $\partial z=x$. Now, by the assumption of disjointness and the 
dimension condition $n=p+q+1$, the intersection $z \cdot y$ is a well-defined zero-chain. Set $L(x, y)=\eta(z \cdot y)$, where $\eta$ is the augmentation (or Kronecker index). To see that $L(x, y)$ is independent of the choice of $z$, suppose $z^{\prime}$ is such that $\partial z^{\prime}=x$, then $z-z^{\prime}$ is a $(p+1)$-cycle and therefore bounds a chain $w$ since $H_{p+1}(M)=0$. Now the intersection $w \cdot y$ is a one-chain and $\partial(w \cdot y)=\partial w \cdot y+w \cdot \partial y=z \cdot y-z^{\prime} \cdot y$, since $y$ is null-homologous. Since the augmentation vanishes on boundaries, $L(x, y)$ is well defined. Note that the above also demonstrates that if $x^{\prime}$ is homologous to $x$ by a chain disjoint from $y$, then $L(x, y)=L\left(x^{\prime}, y\right)$. We briefly note the dual cohomology formulation: if $a$ and $b$ are the Poincaré duals of $x$ and $y$ (say as simplicial chains in a dual triangulation and not just as homology classes for $x$ and $y$ are zero in homology!) and $a=\delta c$ then we may define $L(x, y)=L(a, b)=\langle c \cup$ $b,[M, \partial M]\rangle$, noting that $c \cup b$ is a cocycle by the disjointness condition.

LEMMA 1.2 (PROPERTIES OF LINKING NUMBERS).

(1) Bilinearity: $L\left(a_{1} x_{1}+a_{2} x_{2}, y\right)=a_{1} L\left(x_{1}, y\right)+a_{2} L\left(x_{2}, y\right)$.

(2) (Anti-)Commutativity Law: $L(x, y)=(-1)^{p q+1} L(y, x)$.

(3) Naturality: If $f: M \rightarrow N$ is a homeomorphism then $L\left(f_{*} x, f_{*} y\right)=$ $n L(x, y)$ where $n$ is the degree of $f$.

Proof. Properties 1 and 3 follow easily from corresponding properties of intersections observing that in (3) one needs $f$ to be a homeomorphism (or related assumption) to insure the disjointness of the image cycles. To demonstrate (2), let $x=\partial z_{1}$ and $y=\partial z_{2}$; then by the property of the boundary for intersections,

$$
\partial\left(z \cdot z_{2}\right)=\partial z_{1} \cdot z_{2}+(-1)^{p+1} z_{1} \cdot\left(\partial z_{2}\right)
$$

Since the augmentation vanishes on boundaries,

$$
\begin{aligned}
L(x, y) & =\eta\left(z_{1} \cdot y\right)=\eta\left((-1)^{p+1}\left(\partial z_{1}\right) \cdot z_{2}\right) \\
& =(-1)^{p+1}\left((-1)^{q p+p} z_{2} \cdot \partial z_{1}\right)
\end{aligned}
$$

(by the commutative law for intersections)

$$
=(-1)^{p+1}(-1)^{q p+p} L(y, x)=(-1)^{p q+1} L(y, x) .
$$

Property two is particularly interesting when $p=q$ for then $L$ is a bilinear pairing on the same vector space with a symmetry associated to a manifold of dimension $(n+1)$.

The next proposition plays a crucial role in the proof that our conditions for completing equivariant surgery are necessary.

Proposition 1.3. Let $W^{2 n+2}$ be an oriented manifold with boundary $M$ (oriented coherently) and suppose $H_{p+1}(W)=0$. If $x$ and $y$ are relative cycles of dimension $p+1$ and $q+1$, respectively, and $\partial x$ and $\partial y$ satisfy the conditions for linking in $M$, then $L(\partial x, \partial y)=I(x, y)$ where $I$ is the intersection number in $W$. 


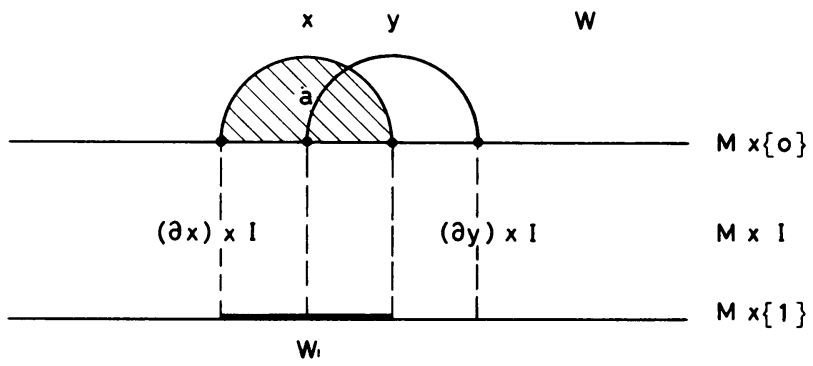

FIGURE 1

Proof. First we note that the hypotheses and conclusion is unchanged if we add a collar $M \times I$ to $W$ along $M$ and replace $x$ by the relative cycle $x_{1}=x+(\partial x) \times I$, and similarly for $y$. By assumption there exists a chain $w_{1}$ in $M \times 1$ with $w_{1}=(\partial x) \times 1$ and $L\left(\partial x_{1}, \partial y_{1}\right)=\eta\left(w_{1} \cdot\left(\partial y_{1}\right)\right)$. Let $w_{0}$ be the corresponding chain in $M \times 0$ and form the $(p+1)$-cycle $z=x-w_{0}$. By assumption $H_{p+1}(W)=0$ so there is a chain $a$ with $\partial a=z$. Since, by construction, $\partial a$ and $\partial y_{1}$ are disjoint (in fact $a$ and $\partial y_{1}$ are) $a \cdot y_{1}$ exists and is a one-chain with boundary

$$
\partial\left(a \cdot y_{1}\right)=\partial a \cdot y_{1} \pm a \cdot \partial y_{1}=\partial a \cdot y_{1}=\left(x-w_{0}\right) \cdot y_{1} \text {. }
$$

Now $w_{0} \cdot y_{1}$ (in $W$ ) $=w_{0} \cdot \partial y$ (in $M \times 0$ ), since $w_{0}$ is carried by $M \times 0$ and intersection commutes with restriction. So, taking augmentations, we have

$$
0=I\left(x, y_{1}\right)-I\left(w_{0}, y_{1}\right)=I(x, y)-L(\partial x, \partial y)
$$

Note that the hypothesis of $H_{p+1}(W)=0$ was crucial to the proof and, in general, some such condition is necessary when $p=q$, since otherwise there may exist $(p+1)$-cycles with nonzero self-intersection number.

The final lemma of this section relates linking numbers to the normal bundle when the cycle is represented by a framed embedded sphere.

LEMMA 1.4 (KERVAIRE-VASQUEZ [KV]). Let $v$ be a nonzero vector field normal to a framed embedded sphere $S^{n}$ in a manifold $M^{2 n+1}$ and let $\bar{S}^{n}$ be the embedded sphere obtained by pushing $S^{n}$ along $v$. If $\Re$ is the complementary bundle to $v$ in the normal bundle of $S^{n}$ in $M$, then $\Re=L\left(S^{n}, \bar{S}^{n}\right) \tau_{n}$ in $\pi_{n}\left(B_{\text {SO(n) }}\right)$ where $\tau_{n}$ is the tangent bundle of $S^{n}$.

Proof. See above.

\section{CHAPTER II: \\ GEOMETRIC KNOT CONCORDANCE THEORY: THE EQUIVARIANT CASE}

1. Preliminaries. We will study the problem of classifying codimension two embeddings of one homotopy lens space in another (which are to be locally flat if one is in the PL or Topological category). As the general classification 
is a very difficult problem, involving all the intricacies and beauty of knot theory, and is unknown, in complete generality, even for codimension two embeddings of spheres in spheres, we will simplify our problem by defining an appropriate equivalence relation: equivariant knot concordance.

We will work in the category of smooth, oriented manifolds.

As in the previous chapter all homology groups will be understood to have integral coefficients. We will perform many of our constructions equivariantly on the covering space, so that it is convenient to consider triples $\left(J^{2 n+1}, K^{2 n-1} ; T\right)$ where $K^{2 n-1}$ is a codimension two homotopy sphere in a homotopy sphere $J$ (with chosen orientations) with $K$ invariant under a smooth fixed point free action of order $m$. Note that these choices are equivalent to defining a "polarization" on the orbit space $Q^{2 n+1}=J / T$ (Wall [W2]).

DEFINITION 2.1. $\left(J, K_{0} ; T\right)$ is equivariantly concordant to $\left(J, K_{1} ; T\right)$ iff there exists an $h$-cobordism $H$ in $J \times I$, invariant under $T \times \operatorname{Id}_{I}$, meeting the boundary transversely and such that $\left(J \times I, H ; T \times \mathrm{Id}_{I}\right) \cap J \times\{i\}$ is diffeomorphic to $\left(J, K_{i} ; T\right)$ for $i=0,1$. This generalizes the usual notion of knot concordance.

The concordance group of knots was first introduced by R. H. Fox and J. Milnor [FM] for the case of $S^{1}$ in $S^{3}$ and eventually computed algebraically in all higher dimensions by Kervaire [K1], [K2], Levine [L4] and Milnor [M4]. We propose to make a similar calculation for equivariant knot concordance theory. Our methods are in the same spirit, as evidenced by our use of linking phenomena, even though the original techniques do not generalize to the equivariant case (see [LdM2]).

Fix an invariant triple $(J, K ; T)$. We will now begin to define the invariants necessary for the classification.

Definition 2.2. A codimension two lens space pair $\left(Q^{2 n+1}, P^{2 n-1}\right)$ is characteristic iff there exists a homotopy equivalence $f: Q \rightarrow L$, a standard lens space, such that $P^{2 n-1}$ is the transverse inverse image of the standard codimension two lens space in $L$.

We first prove a preliminary result necessary in proving that an invariant codimension two sphere is characteristic.

LEMMA 2.3 (COMPARE [Br2]). Let $(J, K ; T)$ be an invariant triple with associated orbit space pair $(Q, P)$. The Euler class of the normal bundle of the inclusion $i: P \rightarrow Q$ is a unit in $H^{2}(P)$.

Proof. We will show this by identifying the Thom isomorphism with the cup product structure in $H^{*}(Q)$. The following diagram commutes by naturality of cup products: 


$$
\begin{array}{ccc}
H^{2}(E, \partial E) \times H^{2 n-2}(E) & \stackrel{\cup}{\rightarrow} & H^{2 n}(E, \partial E) \\
j^{*} \times i^{*} \uparrow & & \cong \uparrow j^{*} \\
H^{2}(Q, Q-\stackrel{\circ}{E}) \times H^{2 n-2}(Q) & \stackrel{\cup}{\rightarrow} & H^{2 n}(Q, Q-\dot{\circ}) \\
k^{*} \times \operatorname{Id} \downarrow \cong & & \cong \downarrow k^{*} \\
H^{2}(Q) \times H^{2 n-2}(Q) & \stackrel{\cup}{\rightarrow} & H^{2 n}(Q)
\end{array}
$$

where $(E, \partial E)$ is the Thom space of the normal bundle identified with the tubular neighborhood pair, $\stackrel{E}{E}=$ interior of $E$ and $j^{*}$ is the excision isomorphism. Now $k^{*}$ is an isomorphism, by the cohomology exact sequence, since $Q-\stackrel{\circ}{E}$ is a (co-)homology circle by Alexander duality. Finally $i^{*}$ is an isomorphism since $E$ retracts to $P$, which is the $(2 n-1)$ skeleton of $Q$.

Now suppose that $w$ in $H^{2}(Q, Q-\stackrel{\circ}{E})$ is such that $j^{*}(w)=U$, the Thom class. Then $w \cup$ is an isomorphism by the upper part of the diagram (the Thom isomorphism). By commutativity of the diagram, $k^{*}(w) \cup$ is also an isomorphism, so that $k^{*}(w)$ must be a unit. Finally,

$$
\text { the Euler class }=j^{*}(U)=i^{*} k^{*}\left(j_{0}^{*-1} U\right)=i^{*} k^{*}(w) . \|
$$

REMARK. By Spanier [Sp, Theorem 17, p. 354], one may show that the Euler class is the Poincare dual of the fundamental class of $\boldsymbol{P}$.

LEMMA 2.4. If $(J, K ; T)$ is an invariant codimension two triple, then $K$ is characteristic (or to be precise $K$ is the cover of a characteristic lens space).

Proof. First note that the normal 2-plane bundle to $P^{2 n-1}$ in $Q$, the orbit spaces, is oriented, hence characterized, by its Euler class. But, by the above lemma, it corresponds to a generator of $H^{2}(P)$. By the homotopy classification of lens spaces, there is a homotopy equivalence $g: P \rightarrow L^{2 n-1}, L$ some standard lens space. Now choose a suspension $L^{2 n+1}$ of $L^{2 n-1}$ (corresponding to the choice of a primitive $m$ th root of unity) so that the Euler class of the normal bundle of $L^{2 n-1}$ corresponds, under $g$, to the above generator of $H^{2}(P)$. We may now extend $g$ to a tubular neighborhood $U$ in $Q$ and $V$ in $L^{2 n+1}$. Now, $L^{2 m+1}-V$ is homotopy equivalent to $S^{1}=K(Z, 1)$ (since in the cover the action on the complement $S^{1} \times D^{2 n}$ is the diagonal action) so by obstruction theory we may extend $g$ to the complement of the tubes iff $g^{*}(i)$ in $H^{1}(U)$ is in the image of $H^{1}(Q-U)$. But, by Alexander duality, the next term in the cohomology exact sequence is zero. Therefore $g$ extends and by construction $g^{-1}\left(L^{2 n-1}\right)=P$.

We now construct a codimension one submanifold in the complement of $K$ in $J$ which plays a very important role. In the classical knot case, this manifold is called the "Seifert surface," for it is two-dimensional oriented manifold bounding a knot $S^{1}$ in $S^{3}$. By Lemma 2.4 we have the following 
diagram:

$$
\begin{array}{ccc}
J / T=Q^{2 n+1} & \stackrel{f}{\rightarrow} & L^{2 n+1} \\
\cup & & \cup \\
K / T=P^{2 n-1} & \stackrel{f}{\rightarrow} & L^{2 n-1}
\end{array}
$$

with $f$ a homotopy equivalence, transverse regular at $L^{2 n-1}$. Since $f$ preserves normal bundles, we may restrict $f$ to the complement of open disc bundles, $X^{*} \subset Q^{2 n+1}$ and $S^{1} \times D^{2 n} \subset L^{2 n+1}$. (The map $f: X^{*} \rightarrow S^{1} \times D^{2 n}$ may easily be made into a degree one normal map [Br4] using trivial bundles.) Let $\bar{V}^{2 n}$ be the transversal inverse image of $D^{2 n}$ in $X^{*}$. Let $X$ be the inverse image of $X^{*}$ in $J$ under the covering projection, $V^{2 n}$, a fixed lift of $\bar{V}$, and $V^{\prime}$, the union of all the (disjoint) lifts. It is interesting to note that we can extend $V^{\prime}$ to a $Z / m Z$ manifold $V^{\prime \prime}$ with Bockstein $K$ (i.e. $K$ is a singularity where $m$ "leaves" meet).

We may orient $V$ so that $\partial_{*}[\bar{V}]=[K]$, the given orientation on $K$. Now $V$ has trivial normal bundle in $X$, which we can choose disjoint from translates and parametrize as $V \times[-1,1]$, so that the preferred orientation agrees with the restriction of the orientation of $J$. We may now define a diffeomorphism $i_{+}: V \rightarrow J$, which pushes $V$ off itself a distance $\varepsilon>0$; an " $\varepsilon$-push" in the positive normal direction.

We are now able to define our linking forms, which will give an a priori definition of the surgery obstruction in our setting. We define integer valued bilinear form $B_{i}(i=0, \ldots, m-1)$ on $H_{n}\left(V^{2 n} ; Z\right)$ by

$$
B_{i}(x, y)=L\left(x, i_{+*}\left(T_{*}^{i} y\right)\right)
$$

where we use the diffeomorphism to make the carriers of $x$ and $y$ disjoint. By Lemma 1.2 and the preceding remark $B_{i}(x, y)$ is well defined on the homology classes of $x$ and $y$ and is bilinear. Note that the $B_{i}$ vanish on the torsion submodule of $H_{n}\left(V^{2 n}\right)$ so that we could just as well define $B_{i}$ on the free module $H_{n}(V) /$ Torsion.

THEOREM $2.5(n>2)$. $\left(J^{2 n+1}, K ; T\right)$ is equivariantly concordant to a trivial invariant knot iff there exists a subspace $H \subset H_{n}(V)$ of one-half the rank on which $B_{i}$ is identically zero $(i=0, \ldots, m-1)$.

REMARKS. (1) This generalizes the theorem of Levine [L3] to the equivariant case.

(2) The algebraic conditions on the forms $B_{i}$ may be weakened (see Corollary 3.9) but this is the most convenient form to use in completing the necessary surgery.

(3) One may prove Theorem 2.5 for $n=2$ using ideas of Wall [W1] and Levine [L3], but the necessary techniques would complicate the presentation. 
(4) While the conditions of the theorem seem to depend on the choice of $V$, the conclusion does not, suggesting that one may intrinsically define the forms $B_{i}$ (see Chapter V).

(5) S. Cappell and J. Shaneson [CS1], [CS2] have also developed techniques for doing surgery in this case and in much greater generality. For the relation between the two methods, see Chapter V. However, using Theorem 2.5, we can compute their algebraic $K$-theoretic obstruction groups in certain cases.

2. Necessity. In this section a strengthened form of necessity for Theorem 2.5 will be shown, by proving that our linking forms are an invariant of equivariant concordance. First, note that reversing the orientation on the ambient sphere $J$ and on $K$ simultaneously reverses the sign of the forms $B_{i}$ (by Lemma 1.2 and observing that the positive normal is unchanged). This motivates

Definition 2.6. The direct sum of linking forms $\left(M_{0}=H_{n}\left(V_{0}\right) ; A_{i}\right)$ and $\left(M_{1} ; B_{i}\right)$ is defined to be $\left(M_{0}+M_{1} ;\left(-A_{i}\right)+B_{i}\right)$.

Since the associated is the empty form if and only if the knot is trivial by the unknotting theorem of Levine [L1] $(n>2)$, necessity for Theorem 2.5 follows from

Proposition 2.7. If $\left(J, K_{0} ; T\right)$ is equivariantly concordant to $\left(J, K_{1} ; T\right)$ then the direct sum of the associated linking forms is equivariantly null-concordant (i.e. $M_{0}+M_{1}$ has a subspace of $\frac{1}{2}$ rank on which $\left(-A_{i}\right)+B_{i}$ is identically zero for $i=0, \ldots, m-1)$.

Proof. Let $H \subset J \times I$ be the invariant $h$-cobordism given by assumption. Then, if $f: Q \rightarrow L^{2 n+1}$ is a homotopy equivalence to a standard lens space, we may arrange that $H / T=\left(f \times \operatorname{Id}_{l}\right)^{-1}\left(L^{2 n-1} \times I\right)$ by Lemma 2.4 since $H / T$ is also an $h$-cobordism. By relative transversality on $f \times \mathrm{Id}_{l}$, we may then pull back a cobordism $Z^{\prime}$ from $\bar{V}_{0}$ to $\bar{V}_{1}$. We can lift $Z^{\prime}$ to a cobordism $Z$ between the lifts $V_{0}$ and $V_{1}$, disjoint from its translates under the free action of $T$. By extending the positive normal to $Z$, one obtains an extension, $j$, to $Z$ of $i_{+}$.

Define $H=\operatorname{ker}\left(H_{n}(\partial Z) \rightarrow H_{n}(Z)\right)$, noting that $H_{n}(\partial Z)=H_{n}\left(V_{0}\right)+$ $H_{n}\left(V_{1}\right)$ since the remainder of $Z$ is an $h$-cobordism of a sphere. By the definition of $H$ we can find relative $(n+1)$-cycles $x$ and $y$ so that $\partial x$ and $\partial y$ are any given elements of $H$. By Lemma 1.3,

$$
B_{i}(\partial x, \partial y)=L\left(\partial x, i_{+*}\left(T_{*}^{i}(y)\right)\right)=I\left(x, j_{*}\left(T_{*}^{i} y\right)\right)=0
$$

since the cycles are disjoint under $j$ and $T^{i}$. So the forms $B_{i}$ vanish simultaneously on $H$. It remains to show that $H$ is of $\frac{1}{2}$ rank in $H_{n}(\partial Z)$ This is the usual argument for computing the index of a boundary for which we use a clever argument due to Kervaire [K2]. By the definition of $H$ we have a long 
exact sequence:

$0 \rightarrow H_{2 n+1}(Z, Z) \rightarrow H_{2 n}(\partial Z) \rightarrow H_{2 n}(Z) \rightarrow \cdots \rightarrow H_{n+1}(Z, \partial Z) \rightarrow H \rightarrow 0$.

Using Lefschetz duality for $(Z, \partial Z)$ and the fact that the rank of the cohomology group is the same as the homology group, we have

$$
\text { rank } H=(-1)^{n} \chi(Z)+\frac{1}{2} \text { rank } H_{n}(\partial Z)+(-1)^{n+1} \frac{1}{2} \chi(\partial Z)
$$

where $\chi$ is the Euler characteristic. But $Z$ is odd dimensional, hence its double has Euler characteristic $0=2 \chi(Z)+\chi(\partial Z)$ by the additivity of $\chi$. Therefore rank $H=\frac{1}{2} \operatorname{rank} H_{n}(\partial Z)$.

The following lemma is needed to show that the null-concordance property is preserved after performing low-dimensional surgery.

Definition 2.8. An integral bilinear form $A$ on a $Z$-module $M$ is an $\varepsilon=( \pm 1)$-form iff $A(x, y)+\varepsilon A(y, x)$ is unimodular.

Now the form $B_{0}$ on $H_{n}(V)$ associated to $(J, K ; T)$ is an $\varepsilon\left(=(-1)^{n}\right)$-form since $B_{0}+\varepsilon B_{0}^{*}$ is the intersection form on $V^{2 n}$ whose boundary is a sphere. To see this, define $i_{-}$in a manner analogous to $i_{+}$using the negative normal direction. Then

$$
\begin{aligned}
B_{0}(x, y)+\varepsilon B_{0}(y, x) & =L\left(x, i_{+*}(y)\right)+\varepsilon L\left(y, i_{+*}(x)\right) \\
= & L\left(x, i_{+*}(y)\right)+\varepsilon L\left(i_{-*}(y), x\right) \text { by the obvious homotopy } \\
= & L\left(x, i_{+*}(y)\right)-L\left(x, i_{-*}(y)\right) \text { by commutativity. }
\end{aligned}
$$

Now the difference $i_{+*}(y)-i_{-*}(y)$ bounds the obvious homotopy whose intersection with $x$ is just the intersection of $x$ and $y$ on $V$, by naturality under restriction. The following lemma will be demonstrated in Chapter III (Lemma 3.3) as its proof is purely algebraic.

Lemma 2.9 (Transitivity). Let $A_{0}$ and $B_{0}$ be $\varepsilon$-forms. Then, if $\left(M_{0} ; A_{i}\right)+$ $\left(M_{1} ; B_{i}\right)$ and $\left(M_{0} ; A_{i}\right)$ are null-concordant so is $\left(M_{1} ; B_{i}\right)$.

3. Sufficiency: Surgery below the middle dimension. We will construct the concordance necessary to complete the proof of Theorem 2.5 by doing framed surgery (in the style of Kervaire and Milnor [KM]) equivariantly on the complement $X$. Note that $X$ is framed since it is the transverse inverse image of $S^{1} \times D^{2 n}$. The following proposition gives necessary conditions on the cobordism $W$ of $X$. Recall that $X$ is $i$-simple if $X \rightarrow S^{1}$ is $i$-connected.

Proposition 2.10. $(n>2)$ If there exists a cobordism $W$ of $X$ to $Y$, an $i$-simple homology circle, with an action $\bar{T}$ extending $T$ on $X$ which is a product on the part of the boundary between $X$ and $S^{1} \times S^{2 n-1}$ (with the product action) and satisfying:

(i) $H_{*}(W)=H_{*}\left(S^{1}\right)$. 
(ii) $\pi_{1}(W)=Z$.

Then $\left(J^{2 n+1}, K ; T\right)$ is equivariantly concordant to an $i$-simple invariant knot.

Note that an $(n+1)$-simple complement $X$ is a trivial knot by Levine [L1].

Proof. By the assumption that $W$ is a product along the appropriate part of $\partial W$, we may "glue" $W$ equivariantly to $D^{2} \times K^{2 n-1} \times I$ by the identity on the boundary. This yields a corbordism $Z$ of $J$ together with an action $T_{1}$ of period $m$, so that one end is $(J, K ; T)$, by construction and the assumption that $\bar{T}$ on $W$ extends $T$ on $X$. The other end is also a homotopy sphere with an invariant codimension two sphere $\left(J_{1}, K_{1} ; T_{1}\right)$ since it is the union of $K \times D^{2}$ and a homology circle and, by construction, is an $i$-simple invariant knot. Next we use the conditions on $W$ to show that $Z$ is an $h$-cobordism. By van Kampen's Theorem,

$$
\pi_{1}(Z)=\pi_{1}\left(D^{2} \times K \times I\right) \underset{\pi_{1}\left(S^{1} \times K \times I\right)}{*} \pi_{1}(W)=1
$$

by condition (ii). By assumption (i) and the Mayer-Vietoris sequence for the excisive triple $\left(Z ; W, D^{2} \times K \times I\right), Z$ is a homology $(2 n+1)$-sphere hence $Z$ is an $h$-cobordism.

By the definition of equivariant concordance, we must produce an $h$ cobordism $L \subset J \times I$ invariant under $T \times \operatorname{Id}_{1}$. Let $\tau$ be the torsion of the orbit $h$-cobordism of $(Z, J)$. Since $n>2$, one can realize $-\tau$ by an $h$-cobor$\operatorname{dism} H$ of $K / T$. Now the boundary of a tubular neighborhood $\nu$ of $K / T$ has $\pi_{1}=Z$ so that the $h$-cobordism induced on the boundary of $r^{*} \nu$ is trivial (where $r$ is the retraction of $H$ to $K / T$ ). By the sum theorem for Whitehead torsion, $Z_{1}=W / T \cup{ }_{\partial \nu \times I} r^{*} \nu$, has torsion zero so, by the $s$-cobordism theorem, is a product. The desired $L$ is $\tilde{H}$, the cover of $H$ in $Z_{1}$.

The first step in the construction of $W$ is to find a framed coborsism $W_{1}$ of $X$ (with an action extending $T$ on $X$ ) to an " $(n-1)$-connected" knot complement (i.e. $\pi_{i}(X)=\pi_{i}\left(S^{1}\right)$ for $i<n$ or, in words, $X$ is a homotopy circle through dimension $(n-1))$. Such knots are often referred to as "simple" knots (not to be confused with the concept of simple homotopy type, etc.). That such a cobordism exists has been shown by Kervaire and UngoedThomas (unpublished) and its relevance to the problem of invariant knots was first recognized by Lopez de Medrano [LdM2], whose work was a starting point for this investigation.

As surgery below the middle dimension is now such a standard procedure, I will only sketch two approaches.

(1) Follow W. Browder's procedure for surgery with $\pi_{1}=Z$ [Br1] by doing framed surgery first on the codimension one submanifold $V$ and then on the complement of $V$, in an equivariant manner. This method has the advantage of increasing the connectivity of $V$ along with that of $X$. 
(2) Use the normal map of the orbit space of $X$ to $S^{1} \times D^{2 n}$ as constructed in $\$ 1$ (with trivial bundles.) Following C. T. C. Wall [W2, Chapter 1], we can make $f(n-1)$-connected modulo boundary via a normal cobordism $W$. Then using Theorem 2 of Levine [L1], we can find a codimension one submanifold $V^{\prime}$ in the result of surgery, $X^{\prime}$, which is $(n-1)$-connected.

The resulting normal cobordisms $W$ of (1) and (2) satisfy conditions (i) and (ii) of Proposition 2.10, for $i=n$. Finally, by Lemmas 2.7 and 2.9 the new $(J, K ; T)$ is still null-concordant.

4. Sufficiency: Completion of surgery. By $\S 3$, we need only prove Theorem 2.5 for triples $(J, K ; T)$ whose invariant complement $X$ is a homotopy circle in dimensions less than $n$ and with the codimension one submanifold $V$ $(n-1)$-connected. Under the assumptions of Theorem 2.5 we will construct a cobordism $W$ with the properties required by Proposition 2.10 in two stages. First, we calculate the (simply-connected) surgery obstruction of $V$, find it is zero and perform equivariant framed surgery on $X$, by means of a cobordism $W$ to $S^{1} \times D^{2 n}$ containing a codimension one cobordism $Z$ of $V$ to $D^{2 n}$. (In terms of the theory of C. T. C. Wall, we show that $\tau(f)$ in $L_{2 n+1}(Z)$ is zero, which is identified with the (simply-connected) obstruction of the codimension one submanifold [Sh2], [Br1].) Then, we equivariantly correct the homology of $W$ to that of a circle. Since surgery on $X$ was performed equivariantly relative to the boundary of $X, W$ now satisfies the conditions of Proposition 2.10.

Step one. Constructing the initial cobordism. As seen in $\$ 2$, the intersection form on $V$ is given by $Q(x, y)=B_{0}+\varepsilon B_{0}^{*}$ (where $B_{0}^{*}$ denotes the transpose) where $B_{0}$ is one of the linking forms defined in $\$ 1$. By the assumption of Theorem $2.5, B_{0}$ vanishes on $H \subset H_{n}(V)$ (which we may assume pure by bilinearity and hence a submodule) which is of $\frac{1}{2}$ rank.

Since $B_{0}$ vanishes on $H$, the intersection pairing does also and therefore in the index case the surgery obstruction on $V$ is zero (see formulation in $[\mathrm{Br} 4$, p. 53]). In the Kervaire Arf invariant case one may identify the quadratic form as $q(x) \equiv B_{0}(x), \bmod 2$, by Lemma 1.4 . So by majority vote since $q=0$ on $H, c(q)=0$.

We will find it convenient to have the following explicit description of the surgery: Represent a basis $x_{i}$ of $H$ by disjoint framed embedded spheres in $V$, thicken trivially in the normal direction to $V$ to obtain framed embeddings of $S^{n} \times D^{n+1}$ in $X$. Use these to perform equivariant framed surgery on $X$ to $X^{\prime}$ with trace $W^{\prime}$. Note that since $V$ is disjoint from its translates we can make the translates of the embeddings disjoint also. Inside $W^{\prime}$ we have a codimension one cobordism $Z^{2 n+1}$ of $V$ to a disc $D^{2 n}$ in $X^{\prime}$, disjoint from its translates. Let $N(D)$ be an open tubular neighborhood of $D$ and its translates 
in $X^{\prime}$. Then $X^{\prime}-N(D)$ has $m$ components permuted by the action (since $D$ separates $X^{\prime}$ ). Let $F_{0}$ be a fundamental domain containing the positive normal to $D^{2 n}$. Since $X$ was a homology circle, the standard Mayer-Vietoris computation of the result of surgery discloses that $H_{n}\left(X^{\prime} ; Z\right)$ is free on the transverse spheres to the added handles and are permuted freely by the action. By excision

$$
H_{k}\left(X^{\prime}, X^{\prime}-N(D)\right)=H_{k}(D \times I, \partial(D \times I))=0 \quad(k>1)
$$

and, therefore, in the homology exact sequence

$$
\begin{aligned}
H_{n-1}\left(X^{\prime}, X^{\prime}-N(D)\right) & \rightarrow H_{n}\left(X^{\prime}-N(D)\right) \stackrel{i}{\rightarrow} H_{n}\left(X^{\prime}\right) \\
& \rightarrow H_{n}\left(X^{\prime}, X^{\prime}-N(D)\right)
\end{aligned}
$$

$i_{*}$ is an isomorphism. Hence $H_{n}\left(F_{0}\right) \subset H_{n}\left(X^{\prime}-N(D)\right)$ is free. Since $F_{0}$ is simply connected, framed and odd dimensional, by Kervaire Milnor [KM, Lemma 5.7] we can complete surgery on a basis of $H_{n}\left(F_{0}\right)$ relative to the boundary $\left(=D^{2 n} \cup K^{2 n-1} \times I \cup D^{2 n}\right)$. By duality, the result of surgery on $F_{0}$ has no homology and is therefore a disc. Transferring the completion of surgery (and framing) equivariantly on $X^{\prime}-N(D)$, we achieve our desired cobordism $W$ of $X$ to $S^{1} \times D^{2 n}$ with an action extending $T$ on $X$ and such that $W$ is framed and $\pi_{1} W=\pi_{1} X=Z$ (by the assumption on the connectivity of $X$ and $n>1$ ).

Step two. Localizing the surgery problem. Let $F$ be a fundamental domain for $W-N(Z)$ (where $N(Z)$ is an open tubular neighborhood of the translates of $Z$ ) containing the positive normal to $Z$. The following observations, together with the hypotheses of Thoerem 2.5 , will enable us to localize the surgery problem to $F$. Let $M$ be the submodule of $H_{n+1}(W)$ described as follows: View the cobordism $W$ in the standard manner as $X \times I \cup$ Handles on $X \times 1$. Let $S_{i}^{n}$ be embedded spheres in $V^{2 n} \subset X \times 0$ representing the homology classes in $H$ on which we performed surgery, with $D_{i}^{n+1}$ the core of the handle attached to $S_{i}^{n} \times 1$. Since $X$ is a homology circle, there exists an $(n+1)$-chain $c_{i}$ in $X \times 0$ which bounds the cycle $\left\{S_{i}^{n}\right\}$. Finally let $y_{i}$ be the homology class of the cycle $\left\{S_{i}^{n} \times I \cup D_{i}^{n+1}\right\}-c_{i}$ (where \{\} denotes associated chain).

LEMMA 2.11. The module $M \subset H_{n+1}(W ; Z)$ spanned by the $y_{i}$ and their translates is a free $Z$-summand of $\frac{1}{2}$ rank.

Proof. Let $W^{\prime}$ be the cobordism resulting from the first stage of surgery in Step $1, W^{\prime}=X \times I \cup_{A} B$ where $A$ is the disjoint union of thickened embedded spheres and $B$, the handles added along $A$. $\left(W^{\prime} ; X \times I, B\right)$ is an excisive triad, so by the Mayer-Vietoris theorem, the following sequence is exact: 


$$
H_{n+1}(X \times I)+H_{n+1}(B) \rightarrow H_{n+1}\left(W^{\prime}\right) \rightarrow H_{n}(A) \rightarrow H_{n}(X \times I)+H_{n}(B)
$$

with the first and last terms zero $(n>1)$ since $X$ is a homology circle and $B$ has contractible components. Hence $H_{n+1}\left(W^{\prime}\right)$ is a free module over the group ring $Z[Z / m Z]$ since $A$ is and, by definition of the Mayer-Vietoris boundary, generated (over $Z[Z / m Z]$ ) by the $y_{i}$. Since surgery on $X^{\prime}$ in stage two of Step 1 was done on free $n$-dimensional classes, which are the boundaries of the Lefschetz duals of the $y_{i}, M$ is free in $H_{n+1}(W ; Z)$ and of $\frac{1}{2}$ rank.

LEMMA 2.12. The ordinary homology intersection form is identically zero on $M$.

Proof. Let $d_{U}$ be the deformation of $W$ which sends $X \times 0$ to $X \times \varepsilon$ in $X \times I \subset W$ and let $d_{+}$be the deformation of $W$ associated to the positive normal field of the union of the translates of $Z$. By bilinearity of the intersection pairing we need only show that it vanishes on the generators of $M$ and by naturality and (anti-)commutativity we may reduce the lemma to computing $y_{i} \cdot\left(T^{j}\right)_{*}\left(y_{k}\right)$. By homotopy invariance this equals

$$
d_{U *}\left(y_{i}\right) \cdot d_{+*}\left(\left(T^{j}\right)_{*} y_{k}\right)=\left(c_{i} \times \varepsilon\right) \cdot i_{+*}\left(T^{j}\right)_{*}\left(x_{k}\right)=L\left(x_{i}, i_{+*}\left(T^{j}\right)_{*}\left(x_{k}\right)\right)
$$

since the other components are disjoint under the deformations. But $x_{i}$ and $x_{k}$ (which are the homology classes of the $S_{i}^{n}$ ) are in $H \subset H_{2 n}(V)$, hence the linking form is zero by the assumption of the theorem.

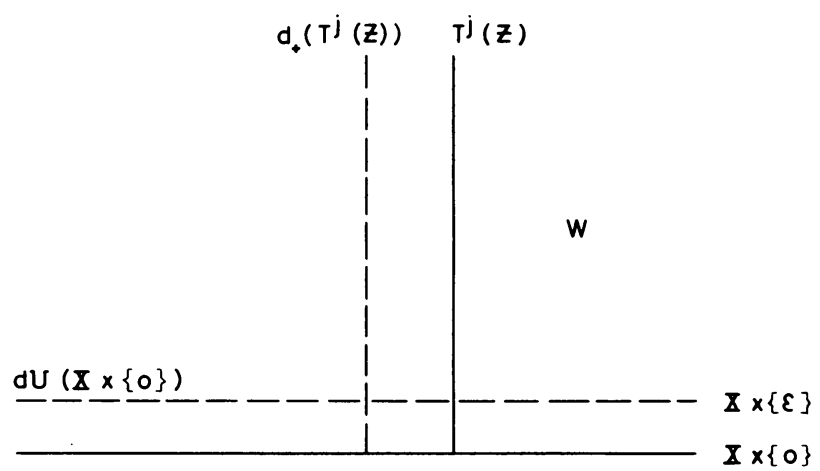

FIGURE 2

LEMMA 2.13. There exists a basis for $M$ as a $Z[Z / m Z]$ module with support in a fundamental domain $F$. 
Proof. Define the map $d_{i}: H_{n}(V) \rightarrow H_{n}\left(T^{i}(V)\right)$ by

$$
z \mapsto I_{+}(X) \stackrel{\partial^{-1}}{\mapsto} c \quad \text { in } C_{n+1}(X) \mapsto c \cdot\left[T^{i}(V)\right]
$$

where $\left[T^{i}(V)\right]$ is the fundamental class, - is the intersection of chains in a manifold $X$ [Lef], and $x$ is a cycle representing $z$. The map defines a cycle (and therefore a homology class) since $c$ and $T^{i}(V)$ have disjoint carriers (by $i_{+}$if $i=0$ and the choice of $V$ disjoint from its translates otherwise). If we made a different choice, $x^{\prime}$, for $x$, then $x^{\prime}$ is homologous to $x$ by a chain $d$ in $V$. If $c$ and $c^{\prime}$ bound $x$ and $x^{\prime}$, respectively, then $c+i_{+}(d)-c^{\prime}$ is a cycle in $X$ and bounds a chain $f$ since $X$ is an homology circle. $f \cdot\left[T^{i}(V)\right]$ is then a homology from $c \cdot\left[T^{i}(V)\right]$ to $c^{\prime} \cdot\left[T^{i}(V)\right]$ since $i_{+}(d)$ is disjoint from $T^{i}(V)$. Similarly $d_{i}$ is independent of the choice of $c$. Note that $d_{j}\left(x_{i}\right)$ is the homological intersection of $y_{i}$ with $T^{j}(V) \subset T^{j}(Z)$ (for $j=0$ the part of $y_{i}$ lying on $Z$ can be trivially made disjoint by the deformation $d_{+}$). In Lemma 2.14, we will show $d_{i}(H) \subset\left(T^{i}\right)_{*}(H)$ where $H$ is the given subspace. Assuming this fact, we complete the proof of 2.13. Since surgery was performed equivariantly,

$$
\left(T^{i}\right)_{*}(H)=\operatorname{ker}\left(H_{n}\left(T^{i}(V)\right) \rightarrow H_{n}\left(T^{i}(Z)\right)\right) .
$$

Therefore since the $x_{i}$ are in $H$, by the claim we can lift the basis $y_{i}$ for $M$ to $z_{i}$ in $H_{n+1}(W-N(Z))$, since $y_{i}$ intersects $Z$ and its translates in zero classes. Clearly $T_{*}$ commutes with the inclusion so we can lift $T_{*} y_{i}$ to $T_{*} z_{i}$. Let $N_{i}$ be the projection of $M$ (lifted) as a $Z$-module to the homology of the $i$ th translate of the fundamental domain $F$. Since $M$ is stable under $T$ (i.e. $T(M) \subset M), T^{j}\left(N_{i}\right) \subset N_{i+j}$. Therefore $N_{i}=T^{i}\left(T^{m-i}\left(N_{i}\right)\right) \subset T^{i}\left(N_{0}\right)$, so $N_{0}$ generates $M$ as a $Z[Z / m Z]$ module.

LEMMA 2.14. $d_{i}(H) \subset\left(T^{i}\right)_{*}(H)$ where $H$ is the given summand of $\frac{1}{2}$ rank in $H_{n}(V)(i=0, \ldots, m-1)$.

Proof. Suppose for some $x$ in $H, d_{i}(x)$ has a nonzero component $z_{1}$ in the complementary summand to $\left(T^{i}\right)_{*}(H)$. Since the intersection pairing on $T^{i}(V)$ is unimodular ( $\partial V$ is a sphere), there is a class $z_{1}^{*}$ dual to $z_{1}$. Since $T_{*}^{i}(H)$ is $\frac{1}{2}$ rank and self-annihilating, we may choose $z_{1}^{*}$ so $z_{1}^{*}=\left(T^{i}\right)_{*}(y)$ for some $y$ in $H$. Now, by the definition of $d_{i}$ and the naturality of intersection under inclusion,

$$
d_{i}(x) \cdot z_{1}^{*}=c \cdot z_{1}^{*}=c \cdot\left(T^{i}\right)_{*}(y)=L\left(x,\left(T^{i}\right)_{*}(y)\right)=0
$$

by the hypothesis of Theorem 2.5, since $x$ and $y$ are in $H$.

But

$$
d_{i}(x) \cdot z_{1}^{*}=\left(z_{0}+z_{1}\right) \cdot z_{1}^{*}=z_{1} \cdot z_{1}^{*}=1
$$


since $\left(T^{i}\right)_{*}(H)$ is self-annihilating under the intersection pairing in $H_{n}\left(T^{i}(V)\right)$ (see Step 1).

Now $M$ is a free summand of $\frac{1}{2}$ rank in $H_{n+1}(W)$ on which the ordinary intersection form vanishes and has a basis over $Z[Z / m Z]$ with support in a fundamental domain $F$ by the preceding lemmas. If we can complete surgery equivariantly on $M$ then the resulting $W^{\prime \prime}$ will be a homology circle and satisfy all the conditions of Proposition 2.10 completing the proof of Theorem 2.5. If $n$ is odd, so $2 n+2$ is divisible by 4 , then since $F^{2 n+2}$ is simply connected and framed we can complete surgery on the basis of $M$ whose support lies in $F$ by standard techniques of framed surgery [KM] since the intersection form is zero on $M$. If $n$ is even then we can perform surgery to a single generator $y$ (over $Z[Z / m Z]$ ) with a single self-intersection (the Kervaire invariant case).

Hence, on the quotient space level, we have a normal cobordism relative to the boundary of our original complement $X$ to $X^{\prime}$ with Kervaire invariant one. By the realization theorem for surgery obstructions, there is a normal cobordism $W^{\prime}$ of $X^{\prime}$ relative to the boundary to a homotopy equivalent space $X^{\prime \prime}$ with Kervaire invariant one. Forming the union of the two normal cobordisms along $X^{\prime}$, we obtain a normal cobordism with surgery obstruction zero, by the additivity property of surgery obstructions. Completing the surgery, we obtain our desired equivariant cobordism to $X^{\prime \prime}$ which is a homotopy circle since $X^{\prime}$ is. This completes the proof.

\section{CHAPTER III: \\ ALGEBRAIC CONCORDANCE THEORY: EQUIVARIANT ISOMETRIC STRUCTURES}

Although the linking forms of Chapter II were the most convenient structure for incorporating the data necessary to complete surgery, they would be very cumbersome in algebraic computations. For this reason we now translate them into an algebraically equivalent but simple setting which was pioneered in the case of ordinary knot concordance by Milnor [M4], Levine [L4] and Kervaire [K1], [K2] (the latter contains an excellent exposition of the algebra).

The basic idea is to change our considerations from a collection of bilinear forms to certain isometries of fixed unimodular forms. Geometrically, the idea originated with the work of John Milnor [M3] (see also Hirzebruch [Hi]) in his discussion of infinite cyclic coverings and their duality properties, However, similar ideas may be found much earlier in the work of Reidemeister [Rei], Burger [Bu] and Blanchfield [Bla]. Algebraically, the idea of isometry is much older and is the basic element of interest in the classical orthogonal groups. 
We model our first definition on the setting used by Kervaire [K2] to study ordinary knot concordance. Fix a positive integer $m$, the order of the free cyclic action, for the remainder of this chapter. Let $R$ be a commutative ring with unit (in this chapter $R$ will either be the integers or the field of rational numbers).

DEFINITION 3.1. An equivariant isometric structure of order $m$ with $\varepsilon$ (= \pm 1 )-symmetry over $R$ will consist of the following (and be denoted $\left.\left(Q ; s_{0}, s_{1}\right)\right)$ :

(i) A free $R$-module $M$ of finite rank together with an $\varepsilon$-symmetric even unimodular bilinear form $Q$ on $M$ (i.e. the adjoint $\operatorname{Ad} Q: M \rightarrow \operatorname{Hom}_{R}(M, R)$ is an isomoprhism and $Q(x, x)$ is even for all $x$ in $M)$.

(ii) A pair of isometries $s_{0}$ and $s_{1}$ of $Q$ : that is $s_{0}$ and $s_{1}$ are in $\operatorname{Hom}_{R}(M, M)$ and satisfy $Q\left(s_{i}(x), y\right)+Q\left(x, s_{i}(y)\right)=Q(x, y)$ for all $x$ and $y$ in $M$ and $i=1,2$.

(iii) $\left\{s_{1}^{m}-\left(s_{1}-\mathrm{Id}\right)^{m}\right\} s_{0}=s_{1}^{m}$ in $\operatorname{Hom}_{R}(M, M)$.

(iv) The minimal polynomial $\phi$ of $s_{0}$ satisfies $\phi(X)=(-\varepsilon)^{h} X^{2 h} \Delta\left(1-X^{-1}\right)$ where $\Delta$ is an even degree $(=2 h)$ reciprocal polynomial over $R$ satisfying $\Delta(1)=(-\varepsilon)^{h}$ and $\Delta(-\varepsilon)$ is an odd square. (A polynomial $\Delta$ is reciprocal provided $\Delta(X)=X^{r} \Delta\left(X^{-1}\right), r=\operatorname{deg} \Delta$.)

One may easily define isomorphism classes of equivariant isometric structures (of the same order $m$ ) in the obvious manner by requiring the $R$-isomorphism of $m$ to preserve the corresponding triples $\left(Q ; s_{0}, s_{1}\right)$. These classes form a monoid under direct sum which we denote $\operatorname{EIS}^{e}(m ; R)$. We now form a group by the following construction.

Definition 3.2. We say ( $Q ; s_{0}, s_{1}$ ) is (equivariantly) concordant to zero (or null concordant) if there is a subspace of $\frac{1}{2}$ the rank of $M$, invariant under $s_{0}$ and $s_{1}$, on which $Q$ vanishes. Furthermore, $\left(Q ; s_{0}, s_{1}\right)$ is (equivariantly) concordant to $\left(P ; t_{0}, t_{1}\right)$ if $\left(Q+-P ; s_{0}+t_{0}, s_{1}+t_{1}\right)$ is concordant to zero.

By the next lemma, transitivity of this relation is verified, reflexiveness and symmetry being trivially satisfied, so that concordance of equivariant isometric structures is an equivalence relation. We denote by $E^{\varepsilon}(m ; R)$ the set of equivalence classes with the group structure induced by direct sum.

LEMMA 3.3. Let $\Phi_{1}$ and $\Phi_{2}$ be equivariant isometric structures with $\Phi_{1}+\Phi_{2}$ and $\Phi_{1}$ null-concordant then $\Phi_{2}$ is null concordant.

Proof. This follows immediately from Kervaire [K2, pp. 87, 94, Remark] and observing that the proof uses only the definition of isometry and the nonsingularity of $Q$.

The proof of Lemma 2.9 is completed by the same appeal, using the bilinearity of each form $B_{i}$.

We now prove the following theorem which provides the translation from 
the geometry of Chapter II to the above algebraic setting.

THEOREM 3.4. Let $\left(J^{2 n+1}, K ; T\right)$ be an invariant knot with associated linking forms $B_{i}$ on $H_{n}\left(V^{2 n}\right), V$ and associated equivariant Seifert manifold. There exists an element $\alpha(J, K ; T)$ in $E^{e}(m ; Z)$ with $\varepsilon=(-1)^{n}$ depending only on the geometric equivariant concordance class of $(J, K ; T)$ such that $\alpha(J, K ; T)=0$ iff the forms $B_{i}$ vanish simultaneously on a subspace of $\frac{1}{2}$ rank of $H_{n}(V)$.

Proof. First we define the equivariant isometric structure $\alpha(J, K ; T)$. Let $M$ be the free $Z$-module, $H_{n}(V ; Z) /$ Torsion, and let $Q(x, y)$ be the intersection form on $M . Q$ is obviously $(-1)^{n}=\varepsilon$ symmetric and unimodular ( $\partial V$ is a sphere). Also $Q$ is even since $V$ is framed, being the transverse inverse image of a point. Now we define the endomorphisms $s_{0}$ and $s_{1}$ of $M$ by the following formulae:

$$
B_{0}(x, y)=Q\left(s_{0}(x), y\right), \quad \sum_{i=0}^{m-1} B_{i}(x, y)=Q\left(s_{1}(x), y\right)
$$

which defines $s_{0}$ and $s_{1}$ since $Q$ is unimodular.

We have previously noted that the intersection form on $H_{n}(V)$ is given in terms of $B_{0}$ by

$$
\begin{aligned}
Q(x, y) & =B_{0}(x, y)+\varepsilon B_{0}(y, x)=Q\left(s_{0} x, y\right)+\varepsilon Q\left(s_{0} y, x\right) \\
& =Q\left(s_{0} x, y\right)+Q\left(x, s_{0} y\right)
\end{aligned}
$$

since $Q$ is $\varepsilon$-symmetric. Therefore $s_{0}$ is an isometry of $Q$. Similarly,

$$
\begin{aligned}
Q\left(s_{1} x, y\right) & +Q\left(x, s_{1} y\right)=Q\left(s_{1} x, y\right)+\varepsilon Q\left(s_{1} y, x\right) \\
& =B_{0}(x, y)+\sum_{i=1}^{m-1} B_{i}(x, y)+\varepsilon B_{0}(y, x)+\sum_{i=1}^{m-1} \varepsilon B_{i}(y, x) .
\end{aligned}
$$

We will show that

$$
\sum_{i=1}^{m-1} B_{i}(x, y)=-\varepsilon \sum_{i=1}^{m-1} B_{i}(y, x)
$$

and conclude that $s_{1}$ is also an isometry. Now

$$
B_{i}(x, y)=L\left(x,\left(T^{i}\right)_{*} y\right)=(-1)^{n+1} L\left(\left(T^{i}\right)_{*} y, x\right)
$$

by the commutative law for linking (Lemma 1.2(2))

$$
\begin{aligned}
& =-\varepsilon L\left(y,\left(T^{m-i}\right)_{*} x\right) \text { by naturality under the homeomorphism } T^{m-i} \\
& =-\varepsilon B_{m-i}(y, x)
\end{aligned}
$$

and $(\dagger)$ follows.

Define $\Delta(X)=\operatorname{det}\left(X B_{0}+B_{0}^{t}\right)$ where $B_{0}$ is (abusing notation) an associated matrix for the form $B_{0}(x, y)$ and ${ }^{t}$ denotes the transpose matrix. The polynomial $\Delta$ is commonly called the Alexander polynomial of the knot 
$(J, K)$. It is well known that $\Delta$ is an even degree $(=2 h)$ reciprocal polynomial satisfying $\Delta(1)=(-\varepsilon)^{h}$ and $\Delta(-\varepsilon)$ is an odd square (Levine [L3]). We relate $\Delta(X)$ to the characteristic polynomial $\rho$ of $s_{0}$ by

$$
\begin{aligned}
(-\varepsilon)^{h} Y^{2 h} \Delta\left(1-Y^{-1}\right) & =(-\varepsilon)^{h} Y^{2 h} \operatorname{det}\left\{\left(1-Y^{-1}\right) B_{0}+\varepsilon B_{0}^{t}\right\} \\
& =(-\varepsilon)^{h} Y^{2 h} \operatorname{det}\left\{\left(1-Y^{-1}-1\right) B_{0}+\left(B_{0}+\varepsilon B_{0}^{t}\right)\right\} \\
& =(-\varepsilon)^{h} Y^{2 h} \operatorname{det}\left\{\left(-Y^{-1}\right) B_{0} Q^{-1}+\operatorname{Id}\right\} \operatorname{det}(Q) \\
& =(-\varepsilon)^{h} \operatorname{det}\left(Y(\text { Id })-B_{0} Q^{-1}\right)(-\varepsilon)^{h}=\rho(Y) .
\end{aligned}
$$

Since the minimal polynomial has the same irreducible factors as the characteristic polynomial [La, p. 402], condition (iv) for an equivariant isometric structure is satisfied.

We now prove the central condition (iii). First we note that

$$
\begin{aligned}
Q\left(s_{1} x, y\right) & =\sum B_{i}(x, y)=\sum L\left(x, i_{+*}\left(T_{*}^{i} y\right)\right) \\
& =L\left(x, i_{+}\left(\operatorname{Id}+T+\cdots+T^{m-1}\right)(y)\right) \\
& =(-1)^{n+1} L\left(i_{+}\left(\operatorname{Id}+\cdots+T^{m-1}\right)(y), x\right)
\end{aligned}
$$

by the additivity and commutativity of linking numbers. But this corresponds to linking $L\left(j_{+*}(y), x\right)$ on $H_{n}\left(V^{2 n}\right)$ in the orbit space $Y=X / T$ since $\left(\mathrm{Id}+T+\cdots+T^{m-1}\right)_{*}(y)$ is invariant under $T_{*}$ and the projection is a local homeomorphism on a neighborhood of $V$.

Let $\tilde{Y}$ be the universal abelian cover of $Y$ corresponding to the subgroup $Z \subset \pi_{1} Y$ generated by the normal circle and let $t$ be the generator of the group of covering translations (in the positive normal direction to $V$ ). Since the action of $T$ on $X$ (the $m$-fold cover of $Y$ corresponding to the map from $Z$ to $Z / m Z$ ) is free, it acts by a primitive $m$ th root of unity on the normal circle to $K$. Therefore there is a least positive integer $r$ whose reduction modulo $m$ is a unit and $T^{r}=\bar{t}$ on $X$ where $\bar{t}$ is induced on $X$ from $t$ on $\tilde{Y}$.

Claim. $B_{i}\left(\left(s_{1}-\mathrm{Id}\right) x, y\right)=B_{i+r}\left(s_{1} x, y\right)$, provided $i+r \neq 0$ (modulo $m$ ). The subscripts on the forms are to be read modulo $m$.

Proof. By the naturality of linking numbers under the homeomorphism $T$, we have

$$
\begin{aligned}
B_{i}\left(\left(s_{1}-\mathrm{Id}\right) x, y\right) & =L\left(\left(s_{1}-\mathrm{Id}\right)(x), i_{+*}\left(T_{*}^{i} y\right)\right) \\
& =L\left(T_{*}^{r}\left(s_{1}-\mathrm{Id}\right)(x), i_{+}\left(T_{*}^{i+r_{y}}\right)\right) .
\end{aligned}
$$

To complete the proof of the claim, we will show that $T_{*}^{r}\left(s_{1}-\right.$ Id $)(x)=\bar{t}_{*}\left(s_{1}\right.$ - Id)(x) is homologous to $s_{1}(x)$ in the complement of $i_{+}\left(T^{i+r}(V)\right)$. In fact we will show that the homology lies in the closure $P$ of the component of $X-T^{i}(V)$ lying between $V$ and $T^{r}(V)=t(V)$. The claim will follow because $i_{+}\left(T^{i+r}(V)\right)$ does not meet this component unless $i+r=0(\bmod m)$ 
and the linking number depends only on the homology class in the complement of $i_{+}\left(T^{i+r}(V)\right)$ (see remark in the paragraph preceding Lemma 1.2).

In $Y$, the orbit space of $X$, let $W$ be the complement of an open tubular neighborhood $N(V)$ of the Seifert manifold $V$. Since $Y$ is a homology circle one may choose bases $\left\{x_{i}\right\}$ for $H_{n}(V)$ and $\left\{y_{i}\right\}$ for $H_{n}(W)$ dually paired by linking (see Levine [L2]), or rather, since $Q$ is unimodular such that $L\left(x_{i}, y_{j}\right)$ $=Q\left(x_{i}, y_{j}\right)$.

Now, as noted above,

$$
L\left(u, j_{+*}(v)\right)=Q\left(s_{1} u, v\right)=Q\left(u,\left(\operatorname{Id}-s_{1}\right) v\right)
$$

by the isometry condition where $u$ and $v$ are in $H_{n}(V)$ and $J_{+}$is the " $\varepsilon$-push" of $V$ in the positive normal direction in $Y$. Therefore the map $\left(j_{+}\right)_{*}$ : $H_{n}(V) \rightarrow H_{n}(W)$ is given by (Id $\left.-\mathrm{s}_{1}\right)$ in terms of the above bases. Likewise, we compute

$$
\begin{aligned}
L\left(u, j_{-*}(v)\right) & =L\left(u, j_{+*}(v)\right)-Q(u, v) \\
& =Q\left(\left(s_{1}-\text { Id }\right) u, v\right)=Q\left(u,-s_{1}(v)\right)
\end{aligned}
$$

hence $\left(j_{-}\right)_{*}=-s_{1}$, where $j_{-}$is the push in the negative normal direction.

The boundary of $W$ has two copies of $V, V_{+}$and $V_{-}$, and in the universal abelian cover (viewed as the union of translates $t^{i}(W)$ ), $t\left(V_{+}\right)$is identified with $V_{-}$. Therefore in $W,\left(T_{*}\right)^{r}\left(s_{1}-\mathrm{Id}\right)(x)=\bar{t}_{*}\left(s_{1}-\mathrm{Id}\right)(x)$ in $V_{-}$is homologous to $\left(j_{-}\right)_{*}\left(s_{1}-\operatorname{Id}\right)(x)=-s_{1}\left(s_{1}-\operatorname{Id}\right)(x)$ in $H_{n}(W)$. Similarly, $s_{1}(x)$ in $V_{+}$is homologous to $\left(j_{+}\right)_{*}\left(s_{1}(x)\right)=\left(\operatorname{Id}-s_{1}\right) s_{1}(x)$ in $H_{n}(W)$.

In case $i+r=0(\bmod m)$ we obtain the following formula:

$$
B_{-r}\left(\left(s_{1}-\text { Id }\right) x, y\right)=B_{0}\left(s_{1} x, y\right)-Q\left(s_{1} x, y\right)
$$

for

$$
L\left(\left(s_{1}-\mathrm{Id}\right) x, i_{+*}\left(T_{*}^{-r}(y)\right)\right)=L\left(T_{*}^{r}\left(s_{1}-\mathrm{Id}\right) x, i_{-*}(y)\right)
$$

because $i_{+*}(y)$ is homologous to $i_{-*}(y)$ in the complement of the support of the first variable. Furthermore, by the above argument, noting that $i_{-}(V)$ does not meet $P$, we have:

$$
=L\left(s_{i} x, i_{-*}(y)\right)=L\left(s_{1} x, i_{+*}(y)\right)-Q\left(s_{1} x, y\right)=B_{0}\left(s_{1} x, y\right)-Q\left(s_{1} x, y\right)
$$

as desired. I wish to thank Chao Chu Liang for alerting me to the distinction of this case.

Next, we need the following polynomial identity for an indeterminate $z$ :

$$
\begin{aligned}
(m>0) \quad z^{m}-(z-1)^{m}= & z^{m-1}+z^{m-2}(z-1)+\cdots \\
& +z(z-1)^{m-2}+(z-1)^{m-1}
\end{aligned}
$$


Proof (Induction). This is trivial for $m=1$ and, by the induction hypothesis,

$$
\begin{aligned}
z^{m}+z^{m-1}(z- & 1)+\cdots+(z-1)^{m-1} \\
& =z\left\{z^{m-1}+z^{m-2}(z-1)+\cdots+(z-1)^{m-1}\right\}+(z-1)^{m} \\
& =z\left\{z^{m}-(z-1)^{m}\right\}+(z-1)^{m}=z^{m+1}-(z+1)^{m+1} .
\end{aligned}
$$

Finally,

$$
\begin{aligned}
Q\left(s_{1}^{m}(x), y\right) & =\sum_{i=0}^{m-1} B_{i}\left(s_{1}^{m-1}(x), y\right) \quad\left(\text { by def }^{n} \text { of } s_{1}\right) \\
= & \left.\sum_{i=0}^{m-1} B_{0}\left(\left(s_{1}-\text { Id }\right)^{i} s_{1}^{m-i-1}(x), y\right) \quad \text { (by Claim and primitivity of } r\right) \\
= & \left.B_{0}\left(\sum_{i=0}^{m-1}\left(s_{1}-\mathrm{Id}\right)^{i} s_{1}^{m-i-1}(x), y\right) \quad \text { (bilinearity of } B_{0}\right) \\
= & B_{0}\left(\left\{s_{1}^{m}-\left(s_{1}-\mathrm{Id}\right)^{m}\right\}(x), y\right) \quad \text { (by the above identity) } \\
= & \left.Q\left(\left\{s_{1}^{m}-\left(s_{1}-\mathrm{Id}\right)^{m}\right\} s_{0}(x), y\right) \quad \text { (by the definition of } s_{0}\right) .
\end{aligned}
$$

Since $Q$ is unimodular and is arbitrary (iii) follows.

So we have defined an equivariant isometric structure $\alpha(J, K ; T)$. $\alpha$ is well defined in $E^{\varepsilon}(m ; Z)$ by the necessary conditions of Theorem 2.5 , which also implies the necessity of the last conclusion.

Now suppose $N$ is a subspace of $H_{n}(V)=M$ given by the null-concordance of $\alpha(J, K ; T)$, i.e. $N$ is of $\frac{1}{2}$ the rank of $M$, self-annihilating under $Q$ and invariant under $s_{0}$ and $s_{1}$. Then, for $x$ and $y$ in $N, B_{0}(x, y)=Q\left(s_{0} x, y\right)=$ 0 since $N$ is invariant under $s_{0}$ and $Q$ vanishes on $N$. Now by the Claim and the primitivity of $r$, as defined in the proof of condition (iii), there is an integer $l$ so that $B_{i}\left(s_{1}^{l} x, y\right)=B_{0}\left(\left(s_{1}-\mathrm{Id}\right)^{l} x, y\right)=0$ since $N$ is a subspace invariant under $s_{1}$ (so also under $\left.\left(s_{1}-\mathrm{Id}\right)^{l}\right)$. So, in particular, $B_{i}$ vanishes on the lattice $L=s_{1}^{l}(N)$ since $y$ is arbitrary. By bilinearity, we may assume that $B_{i}$ vanishes on a direct summand $N_{1}$ in $M$. If $s_{1}$ were injective then $B_{i}$ must vanish on $N$ by rank considerations ( $L$ has the same rank). Lemma 3.5 states that every equivariant isometric structure is concordant to one with $s_{1}$ injective and since algebraic concordance is an equivalence relation we may assume this to complete the proof of the theorem.

The next step in our generalization of Levine's program for computing knot concordance groups is the following lemma asserting the existence of a representative within each concordance class for which the isometries are injective. This is actually a "destabilization" lemma and could be proven purely geometrically for it corresponds to equivariant ambient surgery on trivially linked handles in the Seifert manifold $V^{2 n}$. 
LEMMA 3.5. Given an equivariant isometric structure $\Phi$ there is a concordant structure $\Phi_{1}=\left(Q_{1} ; t_{0}, t_{1}\right)$ with the associated isometry $t_{1}$ injective.

REMARKs (1) $\Phi_{1}$ could be the empty structure.

(2) By condition (iii) in Definition 3.1, $s_{1}$ injective implies that $s_{0}$ is also injective.

Proof. By the isometry condition $Q(x, y)=Q\left(s_{1} x, y\right)+Q\left(x, s_{1} y\right), Q$ vanishes on $L=\operatorname{kernel}\left(s_{1}\right)$. By the unimoduality of $Q$ there exists a subspace $L^{*}$ dually paired to $L$ under $Q$. Let $N$ be the orthogonal complement of $L+L^{*}$ in $M$ under $Q$.

Define $\Phi_{1}=\left(Q_{1} ; t_{0}, t_{1}\right)$ on $N$ by

$Q_{1}=$ restriction of $Q$ to $N$ (it is unimodular by the definiton of $N$ ),

$Q_{1}\left(t_{0} x, y\right)=Q\left(s_{0} x, y\right)$, and

$Q_{1}\left(t_{1} x, y\right)=Q\left(s_{1} x, y\right)$, for $x$ and $y$ in $N$.

One easily checks that this is an equivariant isometric structure on $N$.

Finally we show that $H=L+D=\left\{(l, 0, n, n)\right.$ in $\left.L+L^{*}+N+N\right\}$ in $M+N$ gives a null-concordism of $\Phi+\left(-\Phi_{1}\right)$. By construction $Q+\left(-Q_{1}\right)$ vanishes on $H \times H$ so we need only show invariance under the isometries, $s_{0}+t_{0}$ and $s_{1}+t_{1}$, and it clearly suffices to demonstrate $s_{0}(N) \subset L+N$ and $s_{1}(N) \subset L+N$. But by the definition of isometry,

$$
Q\left(s_{1}(n), l\right)=Q(n, l)-Q\left(n, s_{1}(l)\right)=0-0=0
$$

for $n$ in $N$ and $l$ in $L$, since $L$ is the kernel of $s_{1}$ (and by the definition of $N$ ). Since $l$ was arbitrary in $L, s_{1}(n)$ has no component in $L^{*}$ for all $n$ in $N$. Similarly, since by condition (iii) kernel $s_{0}=L$ also, $s_{0}(N) \subset L+N$.

We are now in a position to reduce the calculation of the group of equivariant isometric structures, $E^{\varepsilon}(m ; Z)$, to the theorem of Levine and Milnor on isometries of bilinear forms. First we observe:

LEMMA 3.6. $0 \rightarrow E^{e}(m ; Z) \rightarrow E^{e}(m ; Q)$ is exact where the second map is given by tensoring with the rational numbers.

Proof. If $Q$ is null concordant, intersect the lattice $M$ in $M \otimes_{z} Q$ with the hypothesized subspace to obtain $L$ in $M$. Trivially $L$ satisfies the conditions for null-concordance in $E^{e}(m ; Z)$.

Now we observe the following in $E^{e}(m ; Q)$ : since we may assume $s_{0}$ and $s_{1}$ are injective by Lemma 3.5 , hence invertible over a field, we may define $S_{0}=\mathrm{Id}-s_{0}^{-1}$ and $S_{1}=\mathrm{Id}-s_{1}^{-1}$. Then, we have

$$
\begin{aligned}
Q\left(S_{0} x, S_{0} y\right) & =Q\left(\left(\mathrm{id}-s_{0}^{-1}\right) x,\left(\mathrm{Id}-s_{0}^{-1}\right) y\right) \\
& =Q(x, y)-Q\left(x, s_{0}^{-1} y\right)-Q\left(s_{0}^{-1} x, y\right)+Q\left(s_{0}^{-1} x, s_{0}^{-1} y\right) .
\end{aligned}
$$

But the isometry condition for the vectors $s_{0}^{-1} x$ and $s_{0}^{-1} y$ and the isometry $s_{0}$ 
implies

$$
Q\left(s_{0}^{-1} x, s_{0}^{-1} y\right)=Q\left(x, s_{0}^{-1} y\right)+Q\left(s_{0}^{-1} x, y\right)
$$

yielding $Q\left(S_{0} x, S_{0} y\right)=Q(x, y)$. (This is the reason for the term isometric structure.) Likewise $Q\left(S_{1} x, S_{1} y\right)=Q(x, y)$. Condition (iii) translates to

$$
\begin{aligned}
S_{0} & =\text { Id }-\left\{\left(s_{1}^{m}-\left(s_{1}-\text { Id }\right)^{m}\right) s_{1}^{-m}\right\} \\
& =\text { Id }- \text { Id }+\left(s_{1}-\text { Id }\right)^{m} s_{1}^{-m}=S_{1}^{m} .
\end{aligned}
$$

Finally we observe that the null-concordance condition translates verbatim for the new automorphisms $S_{0}$ and $S_{1}$. But, since $S_{0}=S_{1}^{m}$, if a subspace is invariant under $S_{1}$, it is trivially so under $S_{0}$, hence the null-concordance class in $E^{\varepsilon}(m ; Q)$ depends only on the isometric structure given by $Q$ and $S_{1}$. We now apply the following theorem of Milnor [M4] and Levine [L4]. (See also the exposition of Kevaire [K2].)

TheOREM 3.7 (LEVINE-MILNOR). Concordance classes of isometric structures over a global field are an infinite sum each of $Z, Z / 2 Z$ and $Z / 4 Z$.

Hence $E^{\varepsilon}(m ; Z)$ is contained in such an abelian group.

COROLlARY 3.8. Theorem 2.5 remains valid assuming the stated conditions on $B_{0}$ and $B_{1}$ only.

Proof. By Theorem 3.4 and Lemma 3.5 we may assume the forms $B_{0}$ and $B_{1}$ are injective. Let $\left(Q ; s_{0}, s_{1}\right)$ be the associated isometric structure (by Theorem 3.4). We will show that the condition on $B_{0}$ and $B_{1}$ implies that $\left(Q ; s_{0}, s_{1}\right)$ is null-concordant, proving the corollary since the condition is logically weaker and therefore necessary (by Theorem 2.5). By Lemma 3.6, we need only show that the rational form is null concordant. Now let $B_{0}$ and $B_{1}$ vanish on a subspace $N$ in $H_{n}(V)$ of one half the rank. As before the condition on $B_{0}$ implies that $N$ is invariant under $s_{0}$, hence also under the rational automorphism $S_{0}=S_{1}^{m}$. By the claim in Theorem 3.4,

$$
B_{1}\left(s_{1}^{r} x, y\right)=B_{0}\left(\left(s_{1}-\mathrm{Id}\right)^{r} x, y\right)
$$

so

$$
B_{1}(x, y)=\left(\mathrm{Id}-s_{1}^{-1}\right)^{r} B_{0}(x, y)=B_{0}\left(S_{1}^{r} x, y\right)
$$

and, therefore, $S_{1}^{r}$ must leave $N$ invariant (since $S_{1}^{m}$ does and $m$ is greater than $r$ ), by the assumption on $B_{1}$. But $r$ is a unit modulo $m$ and $S_{1}$ is an isomorphism over the rational field giving $S_{1}(N) \subset N$.

Geometrically, $S_{0}$ corresponds to the action of the generator of the group of translations on the rational homology of the infinite cyclic covering of $X$, the invariant complement of $K$ in $J$ for the geometric triple $(J, K ; T)$. Now the existence of an action implies the existence of an $m$ th root of $S_{0}$, so that, 
in some sense, this chapter can be constructed as the appropriate setting for lifting the "Galois theory" over the rational field to the integers.

As an immediate consequence of the above results, all elements of the abelian group $E^{\varepsilon}(m ; Z)$ have infinite order or order two or four. By the results of Chapter $\mathrm{V}$ relating our approach to that of $S$. Cappell and $J$. Shaneson, the same is true of the homological surgery group $\Gamma_{2 n}(Z \rightarrow$ $Z / m Z$ ). As the image of the concordance classes of integral equivariant isometric structures in the rational group is unknown (i.e. the cokernel of Lemma 3.6), one must explicitly construct elements to obtain further information on $E^{e}(m ; Z)$. This is the focus of the remainder of Chapter III. We note that any element of $E^{e}(m ; Z)$ occurs for some triple $\left(J^{2 n+1}, K ; T\right)(n>1)$ by the results of Chapter IV.

We now proceed to prove a relation which is crucial to subsequent investigation. First discovered by R. H. Fox in a slightly different setting in his papers on the Free Differential Calculus [F1], where it proved useful in the study of branched cyclic coverings of knots. (Note also the similar equation of Milnor, (*) in Bass [B1], concerning cyclomatic units.)

Let $\left(Q ; s_{0}, s_{1}\right)$ be in $E^{e}(m ; Z)$ with $s_{1}$ injective and $S_{0}$ and $S_{1}$ the associated rational isometries defined previously. Since $S_{0}=S_{1}^{m}$, their characteristic polynomials, $\lambda_{0}$ and $\lambda_{1}$, respectively, are related by

$$
\begin{aligned}
\lambda_{0}\left(X^{m}\right) & =\operatorname{det}\left(X^{m}(\text { Id })-S_{0}\right)=\operatorname{det}\left(X^{m}(\text { Id })-S_{1}^{m}\right) \\
& =\prod_{i=1}^{m} \operatorname{det}\left(X(\text { Id })-\zeta^{i} S_{1}\right)=\prod_{i=1}^{m} \lambda_{1}\left(\zeta^{i} X\right)
\end{aligned}
$$

where $X$ is an indeterminate and $\zeta$ is a primitive $m$ th root of unity.

Proposition 3.9. Let $(Q ; t)$ be an isometry over the integers, with $t$ injective and let $\lambda$ be the characteristic polynomial (over the rational field) of Id $-t^{-1}$. There is a unique rational constant $c$ so that $c \lambda$ is an integral polynomial satisfying $c \lambda(1)=(-\varepsilon)^{n}, \operatorname{deg} \lambda=2 n$.

Proof. The constant is unique, provided it exists, by the normalization condition. Define $L(x, y)=Q(t x, y)$ and let $L$ be an associated matrix. By the isometry condition, $L+\varepsilon L^{t}=Q$. Let $\Delta(X)=\operatorname{det}\left(X L+\varepsilon L^{t}\right)$ which is an integral reciprocal polynomial satisfying $\Delta(1)=(-\varepsilon)^{n} .2 n=\operatorname{rank} L$. Now $S=\mathrm{Id}-t^{-1}=\mathrm{Id}-Q L^{-1}=(L-Q) L^{-1}=-L^{t} L^{-1}$, so that the characteristic polynomial of $S, \lambda(X)=\operatorname{det}\left(L^{-1}\right) \Delta(X)$.

Let $c_{0}$ and $c_{1}$ be the constants furnished by (3.9) for $\lambda_{0}$ and $\lambda_{1}$, respectively. Then:

Proposition 3.10. $c_{0}=c_{1}^{m}$. 
Proof. We will demonstrate that $f\left(X^{m}\right)=\Pi_{i=0}^{m-1} c_{1} \lambda_{1}\left(\zeta^{i} X\right)$ satisfies the conclusion of Proposition 3.9 for $\lambda_{0}$ and the conclusion follows by uniqueness. Let $E=Q(\zeta)$ be the cyclotomic extension of $Q$ by a primitive $m$ th root of one. Now $f\left(X^{m}\right)$, by construction, is a polynomial with coefficients in $Z[\zeta]$. However, $f$ is invariant under the action of the Galois group, hence all its coefficients must lie in $Z$. Furthermore, $f$ is reciprocal since $\lambda_{1}$ is. Now consider the augmentation $Z[\zeta] \rightarrow^{A} Z$ which is a ring homomorphism.

$$
\begin{aligned}
f(1) & =A(f(1))=A\left(\prod_{i=1}^{m} c_{1} \lambda_{1}\left(\zeta^{i}\right)\right)=\prod_{i=1}^{m} A\left(c_{1} \lambda_{1}\left(\zeta^{i}\right)\right) \\
& =\left\{(-\varepsilon)^{n}\right\}^{n}=(-\varepsilon)^{n},
\end{aligned}
$$

by the choice of $c_{1}$.

RemarK. In $E^{e}(m ; Z), c_{0}=\operatorname{det}\left(s_{0}\right), c_{1}=\operatorname{det}\left(s_{1}\right)$, so by condition (iii) and Proposition 3.10, $\operatorname{det}\left\{s_{1}^{m}-\left(s_{1}-\text { Id }\right)^{m}\right\}=1$, i.e. is invertible.

We now begin a series of variations on the theme of

$$
\hat{\Delta}\left(X^{m}\right)=\prod_{i=1}^{m} \Delta\left(\zeta^{i} X\right)
$$

Since $\hat{\Delta}(X)$ is the Alexander polynomial of a knot, i.e. condition (iv), it satisfies $\hat{\Delta}(1)=(-\varepsilon)^{n}$, some $n$, hence

$$
\left|\prod_{i=1}^{m} \Delta\left(\zeta^{i}\right)\right|=1,
$$

i.e. $\Delta\left(\zeta^{i}\right)$ is a unit in the cyclotomic integers $Z[\zeta] \subset Q(\zeta)$. It satisfies the further condition that there exists an automorphism of $Z / m Z$ taking $u=$ $\Delta(\zeta)$ to $u^{-1}$ (See comment of Milnor [M2] that this is true for the generator of $(Z[\zeta])^{*}, \zeta^{5}=1$.) It would be interesting to know if this is true for all cyclotomic units.

Recall the following theorem of Seifert (for the classical knots) and Levine characterizing knot polynomials.

TheOREM 3.11 (SEIFERT [Sei], LeVINe [L3]). There exists an isometry $(Q ; t)$ over the integers with Alexander polynomial $\Delta(X)\left(=(-\varepsilon)^{h} \phi\left(1-X^{-1}\right)\right.$, where $\phi$ is the characteristic polynomial of $t, 2 h=\operatorname{deg} \phi)$ iff

(i) $\Delta(1)=(-\varepsilon)^{h}$,

(ii) $\Delta(X)$ is reciprocal,

(iii) $\Delta(-\varepsilon)$ is a square (redundant if $\varepsilon=-1$ ).

Let $P_{\varepsilon}$ be the set of all integral polynomials satisfying (i), (ii) and (iii) with symmetry $\varepsilon(= \pm 1)$.

There are two special cases of knot polynomials satisfying (*) which we shall consider:

(1) $\Delta(X)=\Delta_{1}\left(X^{m}\right)$ for some knot polynomial $\Delta_{1}$. Note that this equation 
gives a well-defined endomorphism of $P_{\varepsilon}$ (except when $m$ is even, $\operatorname{deg} \Delta_{1} \equiv 2$ (4) and $\varepsilon=+1$, in which case we use $\Delta(X)=(-\varepsilon)^{h(m-1)} \Delta_{1}\left(X^{m}\right), 2 h=$ $\left.\operatorname{deg} \Delta_{1}\right)$.

(b) $\Delta(X)=\Pi\left(\phi_{d}(X)\right)^{n_{d}}$ where $\phi_{d}$ is the $d$ th cyclotomic polynomial ( $\phi$ is of this form for knots occurring in algebraic varieties, e.g. Brieskorn varieties). Note that $\phi_{d}$ is in $P_{\varepsilon}$ (using facts in [La, p. 206], particularly the consequences $\phi_{1}(1)=0, \phi_{d}(1)=d, d$ a prime and $\phi_{d}(1)=1, d$ composite) if $d$ is composite, and if $\varepsilon=+1$ and $d$ is even, then $d / 2$ must be composite also to satisfy condition (iii). In case (a) $\Delta(X)$ satisfies (*) trivially and in case (b) $\Delta(X)$ satisfies (*) according to the following criterion:

LEMMA 3.12. $\phi_{d}(X)$ satisfies (*) with respect to $m$ iff $k=d /(m, d)$ has two distinct prime factors so that $\left(\hat{\phi}_{d}\right)\left(X^{m}\right)=\prod_{i=1}^{m} \phi_{d}\left(\zeta^{i} X\right)=\left\{\phi_{k}\left(X^{m}\right)\right\}^{\phi(d) / \phi(k)}$ (here $\phi$ is the Euler $\phi$-function).

Proof. (Compare Fox [F2] and Gordon [G].)

$$
\begin{aligned}
\prod_{i=1}^{m} \phi_{d}\left(\zeta^{i} X\right) & =\prod_{i=1}^{m} \prod_{j=1}^{\phi(d)}\left(\zeta^{i} X-\omega_{j}\right) \\
& \text { where } \omega_{j} \text { are the primitive } d \text { th roots of unity, } \\
& =\prod_{j=1}^{\phi(d)} \prod_{i=1}^{m}\left(\zeta^{i}\right)^{\phi(d)}\left(X-\zeta^{-i} \omega_{j}\right) \\
& =\prod_{j=1}^{\phi(d)}\left(X^{m}-\left(\omega_{j}\right)^{m}\right)
\end{aligned}
$$

Now $\left(\omega_{j}\right)^{m}$ is a primitive $k=d /(m, d)$ root of unity so $\hat{\phi}_{d}\left(X^{m}\right)$ must be some power of $\phi_{k}\left(X^{m}\right)$ and, by comparing degrees, it must be $\phi(d) / \phi(k)$.

It is curious to note that if $(m, d)=1$, then $\hat{\phi}_{d}=\phi_{d}$.

In case (a) the associated unit in $Z[\zeta]$ is \pm 1 , while in case (b) it is easy to give examples where it is highly nontrivial (e.g. $m=5$, then case (b) for $d=6$ generates $\left.(Z[\zeta])^{*}\right)$.

Finally we note that cases (a) and (b) do not exhaust the possibilities for knot polynomials satisfying (*), for example the following polynomials listed by Gordon [G] for $m=2$,

$$
1-X^{2}+X^{3}-X^{4}+X^{6} \text { and } 1-X^{2}-X^{3}-X^{4}+X^{6} \text {. }
$$

Our second variation occurs in the consideration of the following sequence of groups which is the analog of the inclusion-transfer sequence on the second ring in terms of the Cappell-Shaneson $\Gamma$-groups.

$$
C^{\varepsilon}(Z) \stackrel{i}{\rightarrow} E^{\varepsilon}(m, Z) \stackrel{\operatorname{Tr}}{\rightarrow} C^{\varepsilon}(Z)
$$


where $\operatorname{Tr}\left(Q ; s_{0}, s_{1}\right)=\left(Q ; s_{0}\right)$ and

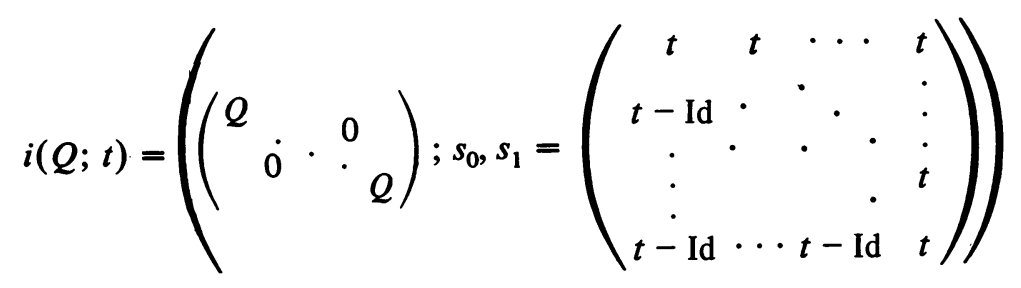

on $m$ copies of $M$.

To show $s_{1}$ is an isometry, we may assume, by bilinearity, that $x$ and $y$ have only one nonzero component, say $x_{i}$ and $y_{j}$. Then

$$
\begin{aligned}
Q_{1}\left(s_{1} x, y\right)+ & Q_{1}\left(x, s_{1} y\right) \\
= & Q_{1}\left(t x_{i}, \ldots, t x_{i},(t-\mathrm{Id}) x_{i}, \ldots,\left(0 \ldots 0, y_{j}, 0 \ldots 0\right)\right) \\
& +Q_{1}\left(\left(0 \ldots 0, x_{i}, 0 \ldots 0\right),\left(t y_{j}, \ldots, y_{j},(t-\mathrm{Id}) y_{j}, \ldots\right)\right) \\
= & \begin{cases}Q(t x, y)+Q(x,(t-\mathrm{Id}) y)=0=Q_{1}(x, y), & i>j, \\
Q\left(t x_{i}, y_{j}\right)+Q\left(x_{i}, t y_{j}\right)=Q\left(x_{i}, y_{j}\right)=Q_{1}(x, y), & i=j, \\
Q\left((t-\mathrm{Id}) x_{i}, y_{j}\right)+Q\left(x_{i}, t y_{j}\right)=0=Q_{1}(x, y), & i<j .\end{cases}
\end{aligned}
$$

We now determine $s_{0}$, hence the composition $\operatorname{Tr}(i)$, and also show that $i$ is well defined. Let $T$ be the matrix

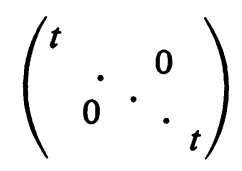

then

Claim. $s_{1}^{m}=T\left\{s_{1}^{m}-\left(s_{1}-\text { Id }\right)^{m}\right\}$.

Proof (INDUCTION). Let $s_{1}^{r}=\left(A_{i j}^{r}\right)$ and $\left(s_{1}-\mathrm{Id}\right)^{r}=\left(B_{i j}^{r}\right)$ in terms of the same blocks as $s_{1}$. From the symmetry of $s_{1}, A_{i j}^{r}$ and $B_{i j}^{r}$ depend only on $l=j-i$, so for convenience, we sometimes denote $A_{i j}^{r}$ by $A_{l}^{r}$, etc. The induction hypotheses are the following symmetry claims:

(i) $\left(t-\right.$ Id) $A_{l+m-r}^{r}=t B_{l}^{r},-m<l<r$,

(ii) $\left(t-\right.$ Id) $A_{l+m}^{r+}=t A_{l}^{r},-m<l<0$,

(iii) $\left(t-\right.$ Id) $B_{l+m}^{r}=t B_{l}^{r},-m<l<0$.

For $r=1$ these claims are obvious. We first demonstrate (iii). 


$$
\begin{aligned}
t B_{l}^{r+1}= & t B_{1-l, 1}^{r+1} \\
= & \sum_{i=1}^{-l}(t-\mathrm{Id}) t B_{i, 1}^{r}+(t-\mathrm{Id}) t B_{1-l, 1}^{r}+\sum_{i=2-l}^{m} t^{2} B_{i, 1}^{r} \\
= & \sum_{i=1}^{-l}(t-\mathrm{Id}) t B_{l+m+i, l+m+1}^{r}+(t-\mathrm{Id})^{2} B_{1, l+m+1}^{r} \\
& +\sum_{i=2-l}^{m} t(t-\mathrm{Id}) B_{l+i, l+m+1}^{r} \\
= & \sum_{i=l+m+1}^{m}(t-\mathrm{Id}) t B_{i, l+m+1}^{r}+(t-\mathrm{Id})^{2} B_{1, l+m+1}^{r} \\
& +\sum_{i=2}^{l+m} t(t-\mathrm{Id}) B_{i, l+m+1}^{r} \\
= & (t-\mathrm{Id}) B_{l+m}^{r+1} .
\end{aligned}
$$

Condition (ii) follows similarly. By (ii) and (iii) it suffices to prove (i) for $l<0$.

$$
t B_{l}^{r+1}=t B_{1-l, 1}^{r+1}=\sum_{i=1}^{1-l}(t-\mathrm{Id}) t B_{i, 1}^{r}+\sum_{i=2-l}^{m} t^{2} B_{i, 1}^{r} .
$$

Assume

$$
\begin{aligned}
l+m+ & r \geqslant 0 \\
= & \sum_{i=1}^{1-l}(t-\mathrm{Id})^{2} A_{m-r-i+1}^{r}+\sum_{i=2-l}^{m} t^{2} A_{m-r-i+1}^{r} \text { by induction on (i) } \\
= & \sum_{i=1}^{1-l}(t-\mathrm{Id})^{2} t A_{-r-i+1}^{r}+\sum_{i=2-l}^{m} t(t-\mathrm{Id}) A_{m-r-i+1}^{r} \text { by (ii) } \\
= & \sum_{i=l+m-1}^{m-1} t(t-\mathrm{Id}) A_{i, m-r-1+l}^{r}+t(t-\mathrm{Id}) A_{m, m-r-1+l}^{r} \\
& +\sum_{i=1}^{m+l-2} t(t-\mathrm{Id}) A_{i, m-r-1+l}^{r} \\
= & (t-\mathrm{Id}) A_{1, m-r-1+l}^{r+1}=(t-\mathrm{Id}) A_{m-(r+1)+l}^{r+1} .
\end{aligned}
$$

Assume

$$
\begin{aligned}
l+m-r< & 0 \\
= & \sum_{i=1}^{m-r}(t-\mathrm{Id})^{2} A_{m-r-i+1}^{r}+\sum_{i=m-r+1}^{1-l}(t-\mathrm{Id})^{2} A_{m-r-i+1}^{r} \\
& +\sum_{i=2-l}^{m} t(t-\mathrm{Id}) A_{m-r-i+1}^{r} \text { by induction on (i) }
\end{aligned}
$$




$$
\begin{aligned}
= & \sum_{i=1}^{m=r}(t-\mathrm{Id}) t A_{-r-i+1}^{r}+"+" \text { by (ii) } \\
= & \sum_{i=r+1}^{m}(t-\mathrm{Id}) t A_{i, 1}^{r}+\sum_{i=1}^{r-m-l+1}(t-\mathrm{Id})^{2} A_{i, 1}^{r} \\
& +\sum_{i=r-m-l+2}^{r} t(t-\mathrm{Id}) A_{i, 1}^{r} \\
= & (t-\mathrm{Id}) A_{r-m-1+2,1}^{r+1}=(t-\mathrm{Id}) A_{m-(r+1)+l}^{r+1} .
\end{aligned}
$$

Condition (i) for $r=m$ is the desired conclusion.

Hence $\hat{\Delta}(X)=\left(\Delta_{t}(X)\right)^{m}$ which implies $\Delta_{s_{1}}(X)=(-\varepsilon)^{n(m-1)} \Delta_{t}\left(X^{m}\right)$. Thus the composition is $m$ times a knot up to isomorphism (and not just concordance class). This is analogous to the corresponding sequence in algebraic $K$-theory (see Milnor [M5] and Bass [B2]).

THEOREM 3.14. $E^{\varepsilon}(m ; Z)$ is infinitely generated over $C^{\varepsilon}(Z)$ with the module structure defined by $i$ and $C^{\varepsilon}(Z)$ is infinitely generated over $E^{\varepsilon}(m ; Z)$.

Proof. The last conclusion follows easily from Proposition 3.10, which implies $\hat{\Delta}(0)=(\Delta(0))^{m}$, hence $\Delta_{s_{0}}(0)$ must be the $m$ th power of an integer. A further necessary condition for $\Phi$ in $C^{e}(Z)$ to be in the image of $\operatorname{Tr}$ is that $\Delta_{t}(X)=\Pi \Delta\left(\zeta^{i} X\right)$ for some $\Delta$ in $P_{\varepsilon}$.

By the previous discussion $\Phi$ in Image of $i$ implies that $\Delta_{s_{1}}(X)=$ $\Delta_{1}\left(X^{m}\right)( \pm 1)$ for some polynomial $\Delta_{1}$ in $P_{\varepsilon}$. Now, given any ordinary isometric structure $\left(Q ; s_{1}\right)$ satisfying $\left|\Pi_{i=1}^{m} \Delta\left(\zeta^{i}\right)\right|=1$, we may associate $\left(Q ;\left\{s_{1}^{m}-\left(s_{1}\right.\right.\right.$ - Id $\left.\left.)^{m}\right\}^{-1} s_{1}^{m}, s_{1}\right)$ in $E^{e}(m ; Z)$ since the condition implies that the expression in brackets is invertible (comment following Proposition 3.10), so we need only find an infinite number of polynomials satisfying (*) not of the form $\Delta\left(X^{m}\right)$. By Lemma 3.12 the $d$ th cyclotomic polynomial satisfies (*) with respect to $m$ iff $k=d /(m, d)$ has two distinct prime factors and $\hat{\phi}_{d}$ is never an $m$ th power so that $\phi_{d} \neq \Delta\left(X^{m}\right)$ for any $\Delta$ in $P_{\varepsilon}$.

Such is the power of the Alexander polynomial.

\section{CHAPTER IV: REALIZATION AND KNOTTED SUSPENSION}

Returning to the geometric situation, we will realize the group $E^{\ell}(m ; Z)$ by geometric triples (as in Chapter II). For convenience we will prove the theorem in the PL (or TOP) category and then discuss the further considerations in the smooth case.

Theorem 4.1 (Realization) ( $n>2$ ). Given a PL fixed point free action $T_{0}$ of period $m$ on a sphere $K^{2 n-1}$ and an element $\beta$ in $E^{\varepsilon}(m ; Z)$, there exists $a$ free action $\left(J^{2 n+1}, T\right)$ on a PL sphere $J$ and an equivariant (locally flat) embedding $\left(K, T_{0}\right) \hookrightarrow(J, T)$ such that $\alpha(J, K ; T)=\beta$. 
REMARKS. (1) Theorem 4.1 actually realizes an element in the monoid of isomorphism classes of equivariant isometric structures.

(2) For $n=2$, in the PL case, one may prove a similar theorem if one restricts $\beta$ to $E_{0}^{\ell}(m ; Z)=\left\{\left(Q ; s_{0}, s_{1}\right) \mid\right.$ signature $\left.Q \equiv 0(16)\right\}$.

Proof. Let $L$ be the orbit space of $K$ and $\xi$ an oriented two-plane bundle on $L$ with Euler class $\chi$, a unit in $H^{2}(L ; Z)=Z / m Z$. Denote by $(D(\xi), S(\xi))$ the associated $\left(D^{2}, S^{1}\right)$ bundle pair. Now in the Gysin sequence for the $S^{1}$ bundle $S(\xi)$ over $L$,

$$
\rightarrow H^{r}(L ; Z) \stackrel{p^{*}}{\rightarrow} H^{r}(S(\xi)) \rightarrow H^{r-1}(L) \stackrel{\cup x}{\rightarrow} H^{r+1}(L) \rightarrow,
$$

the last map is an isomorphism, since $\chi$ is a unit, for $r<2 n-1$. For $r=2 n-1$ and $r=2 n$, we obtain $H^{r}(S(\xi))=Z$. Hence $S(\xi)$ is a homology $S^{1} \times S^{2 n-1}$ and by the homotopy exact sequence, a homotopy product, hence standard in the PL category.

Let $\beta$ in $E^{\varepsilon}(m ; Z)$ be given by $\left(Q ; s_{0}, s_{1}\right)$. In our setting we have the following translation of a theorem of Kervaire [K1]:

TheOREM 4.2 (KeRVAIRE). There exists a simple knot complement $(n>2)$ $\left(X^{2 n+1}, \partial X\right)\left(\right.$ i.e. $X$ is a homology circle with boundary a homotopy $S^{1} \times S^{2 n-1}$ and $\pi_{i}(X)=\pi_{i}\left(S^{1}\right)$ for $\left.i<n\right)$ with associated isometry $\left(Q ; s_{1}\right)$ in $C^{e}(Z)$.

Proof (SkeTch). $Q$ determines the intersection form on the Seifert manifold $V^{2 n}$, which may be chosen to be an $(n-1)$-connected handlebody. By Smale-Hirsch immersion theory we may immerse $V$ in $S^{2 n+1}$. By general position we obtain an embedding on the $n$-skeleton of $V$ and hence can embed $V^{2 n}$ in a small tube about the $n$-skeleton. Using the Seifert linking matrix $A$ associated to $\left(Q ; s_{1}\right)$ by $A(x, y)=Q\left(s_{1} x, y\right)$, one corrects the linking on $H_{n}(V)$ to that given by $A$. Assuming $n>2$ and $Q$ unimodular implies that $\partial V$ is a sphere, so removing a tubular neighborhood of $\partial V$ we obtain our desired knot complement $X$.

So there exists $(X, \partial X)$ realizing $\left(Q ; s_{1}\right)$. Note that $s_{1}$ determines $s_{0}$ by condition (iii) in Definition 3.1.

Define $M=(D(\xi), S(\xi)) \cup_{S^{1} \times S^{2 n-1}}(X, \partial X)$ by identifying the boundaries. Since $X$ is simple and $n>1, \pi_{1} X=Z$ and, by van Kampen, $\pi_{1} / M=$ $Z / m Z$. Let $J=\tilde{M}$, the universal cover of $M$ which we may decompose as $K \times D^{2} \cup_{S^{1} \times S^{2 n-1}} \hat{X}$, where $\hat{X}$ is the $m$-fold cover of $X$ corresponding to $\pi_{1} X=Z \rightarrow Z / m Z$.

Let $\tilde{X}$ be the universal cover of $X$. Since $X$ is simple, $H_{n}(\tilde{X} ; Z)$ is the only nonzero group (Milnor [M3]). Now $\tilde{X}$ is also the universal cover of $\hat{X}$ with the generator of the group of covering translations corresponding to $t^{m}, t$ being the generator for $\tilde{X} \rightarrow X$. By the proof of Propositions 3.9 and 3.10, the Alexander polynomial $\hat{\Delta}(X)$ associated to the isometry for $\tilde{X} \rightarrow \hat{X}$ satisfies 
$\hat{\Delta}\left(X^{m}\right)=\Pi_{i=1}^{m} \Delta\left(\zeta^{i} X\right)$, where $\zeta$ is a primitive $m$ th root of unity and $\Delta$ is the Alexander polynomial for $\tilde{X} \rightarrow X$ (and $s_{1}$ ). By assumption, $\beta$ is in $E^{e}(m ; Z$ ) so that $\hat{\Delta}(1)= \pm 1$ (condition (iv) of Definition 3.1). By Levine [L2], the Alexander polynomial is the determinant for a presentation of $H_{n}(\tilde{X} ; Z)$ over $Z\left[t^{m}, t^{-m}\right]$, and since $H_{n}(\hat{X} ; Z)$ is the cokernel of the presentation under the augmentation $\left(t^{m} \rightarrow 1\right)$, the polynomial condition implies that $X$ is also a homology circle.

Therefore, by the Mayer-Vietoris sequence, $J=\tilde{M}$ is a homotopy sphere, hence PL standard. This defines $\left(J^{2 n+1}, T\right)$ so that $\alpha(J, K ; T)=\beta$.

The construction of the theorem will be referred to as knotted suspension (by the knot and the Euler class). Note that in the PL case Theorem 2.5 is equivalent to

TheOREM 2.5 (PL, $n>2)$. $\left(J^{2 n+1}, K ; T\right)$ is equivalently concordant to a standard suspension $S^{1} * K$ iff $\alpha(J, K ; T)=0$, since PL homotopy $S^{1} \times S^{2 n-1}$ are standard.

We now wish to investigate how "knotted suspension" affects the action. By the work of Browder, Petrie and Wall [BPW] (if $n$ is odd or two) $(J, T)$ is classified by two invariants $\delta$, a torsion invariant and $\rho$, the multisignature. In the following lemma we compute $\delta$ for $(J, T)$ from $\delta$ for $\left(K, T_{0}\right)$ and invariants of the knot and normal bundle. For $\rho$, so far, we have only the theoretical computation given in Chapter $\mathrm{V}$.

LEMMA 4.3. $\delta(J, T)=\left[\left(\zeta^{x}-1\right) / \Delta\left(\zeta^{x}\right)\right] \delta\left(K, T_{0}\right)$ in $Q(\zeta)$ where $\Delta$ is the Alexander polynomial of $s_{1}$ for $\beta=\left(Q ; s_{0}, s_{1}\right)$ and $\chi$ is viewed as an element in $Z / m Z\left(=H^{2}\left(K / T_{0} ; Z\right)\right.$ with generator $\left.T_{0}\right)$ determined by the normal bundle to $K / T_{0}$.

Proof. Use the equivariant (cellular) decomposition of $J$ given by $K \times D^{2}$ $\cup_{S^{1} \times S^{2 n-1}} X$. The torsion $\delta$ is as defined for "special" complexes by Milnor in [M2]. First, as in Wall [W2, 14E.8], one notes there is an exact sequence of equivariant chain complexes:

$$
0 \rightarrow C_{*}(X)+C_{*}(D(\xi)) \rightarrow C_{*}(J) \rightarrow C_{*}\left(S^{1} \times K\right) \rightarrow 0 .
$$

Now $C_{*}\left(S^{1} \times K\right)$ is the tensor product of equivariant chain complexes with the diagonal action, so by naturality has torsion zero, since the product complex with the product action does by the product theorem, as the Euler characteristic of $S^{1}$ is zero. Also $D(\xi)$ collapses to $K$ and so has torsion $\delta\left(K, T_{0}\right)$.

Next, we find the torsion for the homology circle $\hat{X}$. Viewing $\hat{X}$ as the $m$-fold cover of $X$, with $\tilde{X} \rightarrow X$ its universal cover, $\left.T\right|_{\hat{X}}$ corresponds to $t$ where $t$ is the generator of the group of covering translations of $\tilde{X}$ (uniquely determined if one considers orientations). Now Milnor [M1], [M3, Remark, p. 
121] has computed the Reidemeister torsion of $X$ over $Q(z)$, the field of rational functions, to be $(z-1) / \Delta(z)$ with $\Delta=$ Alexander polynomial for $X$, a simple knot complement (in fact, [M3] extends this to any knot complement). Now, watching identifications, $C_{*}(X)$ as a $Q(\zeta)$ module is just $C_{*}(X)$ under the map $z \rightarrow \zeta^{x}$ and the result follows.

In the smooth category, one encounters the usual problem of smoothness structure. If $(J, K ; T)$ is a smooth invariant triple, then in $J / T$, the boundary of a tubular neighborhood of $K / T$ is a homotopy $S^{1} \times S^{2 n-1}$, as in Theorem 4.1, and so has a smoothing given by

$$
Y=\left(S^{1} \times S_{0}^{2 n-1}\right) \# \Sigma_{1}^{2 n}
$$

by [Sch] where $S_{0}$ is a smoothing of $S^{2 n-1}$ and $\Sigma_{1}^{2 n}$ of $S^{2 n}$. Now, $Y=X$, a knot complement, which can be framed (as it is the transverse pullback of the trivial knot complement since any codimension two lens space is characteristic), so $S_{0}^{2 n-1}$ is the boundary of the framed Seifert manifold, and $\Sigma_{1}^{2 n}$ must also bound a framed manifold (since $\Sigma_{1}^{2 n}$ is the boundary of the framed manifold obtained by doing framed surgery on the generator of $H_{1}(Y)$; see Schultz [Sch, Theorem 1.2]). So $\Sigma_{1}^{2 n}$ is standard since $\theta^{2 n}(\partial \pi)=0$ by [KM]. Now the structure on $S_{0}^{2 n-1}$ is determined by $\alpha(J, K ; T)=\left(Q ; s_{0}, s_{1}\right)$ as follows in terms of the bounding Seifert manifold $V^{2 n}$ :

(i) $n \equiv 0$ (4), signature $V=$ signature $Q$.

(ii) $n \equiv 2$ (4), Kervaire Arf $V={ }_{1}^{0}$ according as $\Delta(1) \Delta(-1) \equiv \begin{gathered} \pm 1 \\ \pm 3\end{gathered}$ (8) by Levine [L2] and Robertello [Ro].

So, given a smooth action $\left(K, T_{0}\right)$, if the smoothness structure on $S(\xi), \xi$ a 2-plane bundle over $K / T_{0}$, is of the form $S^{1} \times S_{0}^{2 n-1}$ for $S_{0}^{2 n-1}$ in $\theta^{2 n-1}(\partial \pi)$ and $\alpha$ satisfies the above condition with respect to $S_{0}$, then the smooth knotted suspension may be formed. Note, however, that it is not unique, because of the choice of the gluing diffeomorphism.

One may define a group operation in the geometric theory of equivariant concordance of invariant knots which reflects the algebraic group structure. The definition is confined to the PL or TOP category for reasons which will become apparent. We further restrict our universe to the set of equivariant concordance classes of (PL or TOP, locally flat) invariant triples $\left(J^{2 n+1}\right.$, $\left.K^{2 n-1} ; T\right)$, with the action $(K, T)$ equivalent to a fixed action $\tau$ of order $m$ and with the Euler class of the normal 2-plane bundle of $K / T$ given by $\chi\{T\}$, where $\{T\}$ is the generator of $H^{2}(K / T ; Z)$ corresponding to the action and $\chi$ is a residue modulo $m$. This set will be denoted $E C_{m}^{2 n+1}(\tau ; \chi)$.

Definition 4.4. The composition, $\square$, of two elements in $E C(\tau ; \chi)$ is defined as follows:

Let $\left(J_{1}, K ; T_{1}\right)$ and $\left(J_{2}, K ; T_{2}\right)$ be triples representing the given classes and let $X_{1}$ and $X_{2}$ be the respective invariant closed complements of $K$, with orbit space $Y_{i}, i=1,2$. Let $Y=Y_{1} \#_{Z} Y_{2}$ be the connected sum over the normal 
circle constructed in the following manner: In the PL category, $\partial Y_{i} \cong S^{1} \times$ $S^{2 n-1}=R$. Choose a fixed identification of $R$ with $S^{1} \times E_{+}^{2 n-1} \cup S^{1} \times$ $E_{-}^{2 n-1}$ (with boundaries identified and $E_{ \pm}$two hemispheres whose union is $\left.S^{2 n-1}\right)$. Then $Y=Y_{1} \cup_{S^{1} \times E_{-}} Y_{2}$ and $\partial Y \cong R$. Let $D(\xi)$ be the normal $D^{2}$-bundle to $K / T$. Define a $P L$ manifold $M=Y \cup_{R} D(\xi)$ by identifying the boundaries. Finally let $\left(J_{1}, K ; T_{1}\right) \square\left(J_{2}, K ; T_{2}\right)=(\tilde{M}, K ; t)$ where $t$ is the generator of the group of covering translations of the universal cover, $\tilde{M}$, of $M$ corresponding to $T_{i} \mid K$. This is an invariant triple by the now standard Mayer-Vietoris argument since the $m$-fold cover of $Y$ is a homology circle as the $Y_{i}$ satisfy this condition. The assumption that the Euler classes were equivalent was implicitly used to coherently identify the actions on $X_{1}$ and $X_{2}$ in $\tilde{M}$. A relative version of the construction shows that the operation is well defined on equivariant concordance classes. This operation is easily seen to give a commutative semigroup structure to $E C(\tau ; \chi)$ with identity given by the suspension, $S^{1} * K$, of $K$ determined by $\chi$. The existence of an inverse follows from Theorem 2.5 since $(J, K ; T) \square(-J,-K ; T)$ has linking invariant $\left(M \oplus M ; A_{i} \oplus\left(-A_{i}\right)\right)$ and the diagonal $H=\{(x, x): x$ in $M)$ is the required subspace of one-half rank. Note that the Seifert manifold for the composition is the connected sum along the boundary of the individual Seifert manifolds. From this observation it follows that the map $\alpha$ of Theorem 3.4 is a group homomorphism. It also clarifies the necessity of the restriction to the PL category unless $K$ has the standard differentiable structure.

THEOREM 4.5 (PL, $n \geqslant 3) . E C_{m}^{2 n+1}(\tau ; \chi) \stackrel{\alpha}{\rightarrow} E^{e}(m ; Z)$ is an isomorphism of groups.

Proof. The above discussion implies that $\alpha$ is a well-defined group homomorphism. By Theorem 4.1, $\alpha$ is a surjection and by Theorem 2.5 (see also the discussion following Theorem 4.1), the kernel is the identity.

In other words, if two invariant triples with corresponding Euler classes and PL equivalent actions on the invariant spheres, have the same $\alpha$ invariant, they are PL equivariantly concordant. These conditions are also necessary (since equivariant concordance implicitly assumes the same ambient action). In the smooth category, the same proof demonstrates that $\alpha$ is an injection, if $K$ is the standard sphere.

\section{CHAPTER V: CONNECTIONS}

A primary source of inspiration for this paper was the intriguing results of Santiago Lopez de Medrano [LdM3] concerning codimension two spheres invariant under free involutions on homotopy spheres. In this first section we will highlight the relationships between our methods and those used by Lopez de Medrano.

Returning to the setting of Chapter II, the objects of interest will be triples 
$\left(J^{2 n+1}, K ; T\right)$ where $T$ is a fixed point free involution $\left(T^{2}=\mathrm{Id}\right)$. First we observe that the linking form $B_{1}(x, y)$ defined on $H_{n}\left(V^{2 n}\right), V$ an equivariant Seifert manifold, is $(-1)^{n+1}$-symmetric, since

$$
\begin{aligned}
B_{1}(y, x) & =L\left(y, T_{*} x\right)=L\left(T_{*} y,\left(T_{*}\right)^{2} x\right)=(-1)^{n+1} L\left(x, T_{*} y\right) \\
& =(-1)^{n+1} B_{1}(x, y)
\end{aligned}
$$

by the symmetry law for linking numbers, noting that $T_{*}$ preserves orientations. Even though the integer valued form $B_{1}$ is not unimodular, we can relate the desuspension invariant $\sigma\left(J^{4 l+3}, T\right)$ of Browder and Livesay [BL] to the signature of $B$ when $n$ is odd. In fact,

Proposition 5.1. $\sigma\left(J^{4 l+3}, T\right)=\operatorname{signature}\left(B_{1}\right)$ and is the index of the orbit knot complement.

Proof. Since $\sigma(J, T)$ is independent of the choice of the characteristic codimension one submanifold $W$, we will choose $W=V \cup(-T(V))$ where $V$ is an (oriented) equivariant Seifert manifold associated to $(J, K ; T)$. Note that $T(V)$ is given the opposite orientation so that $W$ has a coherent orientation. In order to compute the desuspension invariant we will produce a map

$$
\text { Ф: } \begin{aligned}
H_{n}(W) & =H_{n}(V)+H_{n}(T(V)) \\
& \rightarrow \operatorname{Kernel}\left(i_{*}: H_{n}(W) \rightarrow H_{n}(A)+T_{*} \operatorname{Ker}\right),
\end{aligned}
$$

where $A$ is the component of $J-W$ "containing" the positive normal of $W$.

First we compute the linking form $L\left(x, i_{+*}(y)\right)$ on $K+T_{*} K$ where $K=$ $\operatorname{Kernel}\left(i_{*}\right)$ and $i_{+}$is as usual (see also [LdM2]). We can find a basis for $K$ and $T_{*} K$ so that the intersection form $I(x, y)$ is given by

$$
\left(\begin{array}{cc}
0 & I \\
\varepsilon I & 0
\end{array}\right)
$$

since the intersection form is unimodular and it must vanish on $K$. Now for $x$ in $H_{n}(W), k$ in $K$, we have $L\left(x, i_{+*}(k)\right)= \pm L\left(i_{+*}(k), x\right)=0$ since $i_{+*}(k)$ bounds a chain in $A$ disjoint from $W$ (which carries $x$ ). Even more trivially, $L\left(p, i_{+*}(x)\right)=0$ for $p$ in $T_{*} K$. So we need only find $L\left(k, i_{+*}(p)\right)=I(k, p)$ $\pm L\left(p, i_{+*}(k)\right)=I(k, p)$, by the standard relation between linking numbers on $W$ and the intersection form (see 2.3). So, with respect to the chosen bases, $L\left(x, i_{+*}(y)\right)$ is given by $\left(\begin{array}{l}0 \\ 0\end{array}\right)$ on $K+T_{*} K$. With respect to the decomposition of $H_{n}(W)=H_{n}(V)+H_{n}(T(V)), L\left(x, i_{+*}(y)\right)$ is given by

$$
\left(\begin{array}{cc}
B_{0} & B_{1} \\
B_{1} & -\varepsilon B_{0}^{t}
\end{array}\right),
$$


where $B_{0}$ is the associated matrix for $B_{0}(x, y)$ and $H_{n}(T(V))$ is given the corresponding basis under $T_{*}$.

We now produce the desired transformation of linking forms noting first that another choice would give us an automorphism of $\left(\begin{array}{ll}0 & I \\ 0 & 0\end{array}\right)$ which must be of the form

$$
\left(\begin{array}{cc}
P & 0 \\
0 & P^{-t}
\end{array}\right)
$$

This automorphism simply conjugates the Browder-Livesay form $x \cdot T_{*} y$ leaving the signature invariant.

Claim.

$$
\Phi=\left(\begin{array}{cc}
Y-\text { Id } & -Y \\
-\varepsilon Q^{-1} S^{-1} Y & \varepsilon Q^{-1} S^{-1}(Y-\text { Id })
\end{array}\right)
$$

is one choice, where $Q=$ intersection matrix on $H_{n}(V), Y=$ matrix associated to $s_{1}$ of the equivariant isometric structure, $S=Y^{2}-(Y-I d)^{2}$ which is invertible by the remark following Proposition 3.10.

Proof. First, one checks that

$$
\Phi^{-1}=\left(\begin{array}{cc}
(\mathrm{Id}-Y) S^{-1} & -\varepsilon Y Q \\
-Y S^{-1} & \varepsilon(\mathrm{Id}-Y) Q
\end{array}\right)
$$

so that

$$
\Phi^{-1}\left(\begin{array}{cc}
0 & \mathrm{Id} \\
0 & 0
\end{array}\right) \Phi^{-t}=\left(\begin{array}{cc}
(\mathrm{Id}-Y) S^{-1}(\mathrm{Id}-Y) Q & (\mathrm{Id}-Y) S^{-1} Y Q \\
Y S^{-1}(\mathrm{Id}-Y) Q & Y S^{-1} Y Q
\end{array}\right)
$$

which by condition (iii) of 3.1 and the claim in Thoerem 3.4 is $L\left(x, i_{+*}(y)\right)$ as computed above. (Note that the isometry condition implies $Y^{t}=Q^{-1}(\mathrm{Id}-$ Y)Q.)

Noting that the intersection matrix on the basis for $K+T_{*} K$ is $\left(\begin{array}{ll}\text { Id } \\ 0\end{array}\right)$ we need only compute $T_{*}$ with respect to this basis to determine $x \cdot T_{*} y$. But

$$
T_{*}=\Phi\left(\begin{array}{cc}
0 & \text { Id } \\
\text { Id } & 0
\end{array}\right) \Phi^{-1}
$$

since $T_{*}$ simply interchanges the Seifert manifolds. Hence

$$
T_{*}=\left(\begin{array}{cc}
0 & \varepsilon S Q \\
-(S Q)^{t} & 0
\end{array}\right)
$$

But $Y=C Q^{-1}$ where $C$ is a Seifert matrix for the orbit knot complement, so

$$
\varepsilon S Q=\varepsilon(\operatorname{Id}-2 Y) Q=\varepsilon(Q-2 C)=-\varepsilon\left(C-\varepsilon C^{t}\right),
$$

which is just the index of the orbit knot. Finally $B_{1}=Y S^{-1}(\mathrm{Id}-Y) Q=$ $C(\varepsilon S Q)^{-1} C^{t}$ has the same signature since signature is a conjugacy invariant.

In case $n$ is even, one may also obtain the following, first demonstrated by 
Lopez de Medrano [LdM1] is his thesis using Browder-Livesay desuspension invariants:

Proposition 5.2. $\left(J^{4 l+1}, T\right)$ admits an invariant knot iff $c(J, T)=0$, i.e. it desuspends.

The following is a most intriguing question of Lopez de Medrano [LdM1]: Does there exist a null-concordant invariant knot with desuspension invariant $\equiv 8(\bmod 16) ?$

This may be reduced to the following realization problem in algebraic knot concordance theory:

Problem 1. Does there exist an isometry $(Q, \hat{s})$ with $\Delta_{s}(X)=\Delta_{1}\left(X^{2}\right)$ (for some polynomial $\left.\Delta_{1}\right)$ and with signiture $\equiv 8(16)$ ?

One can show that knotted suspension with such a knot for $m=2$ is algebraically concordant to $2 a$, for some $a$ in $C^{\varepsilon}(Q)$ :

Problem 2. Is $a$ realized by an integral isometry?

An affirmative solution to Problems 1 and 2 has been found and will appear in a paper by the author entitled Non-injectivity of the transfer of skew-Hermitian forms with applications to topology.

We now commence discussion of the relation between the viewpoint taken in this work and the algebraic $K$-theoretic obstruction group defined and used by Sylvain Cappell and Julius Shaneson in their magnum opus [CS2] on abstract codimension two surgery.

In the rest of this section, we will use freely the notation of [CS2]. It will be necessary to consider the following diagram of rings:

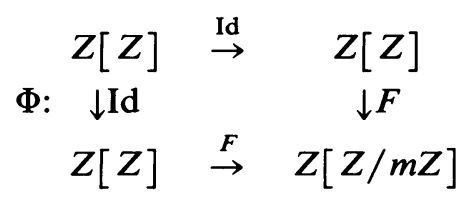

where $F$ is the natural projection. We now restate Proposition 10.2 of [CS2]:

Proposition 5.3. The action of $\Gamma_{2 k+2}^{h}(\Phi)$ on the homological surgery structure set $S_{F}\left(D^{2 k} \times S^{1}\right)$ is transitive and free for $k>2$.

Note that all "structures" are normally cobordant since $\pi_{1}(G / 0)=$ $\pi(G / \mathrm{PL})=0$. By Theorems 2.5, 3.4 and 4.1 (see also Proposition 2.10) we may identify $S_{\Gamma}\left(D^{2 k} \times S^{1}\right)$ with $E^{\varepsilon}(m ; Z), \varepsilon=(-1)^{k}$. So we obtain an isomorphism of pointed sets:

Proposition 5.4. $\Gamma_{2 k+2}^{h}(\Phi)=E^{\varepsilon}(m ; Z), \varepsilon=(-1)^{k}$.

Similarly, using Proposition 10.1 of [CS2], one can relate $E^{\varepsilon}(m ; Z)$ geometrically with $C(-, \tau)=\cup_{\rho} C(\rho, \tau)=$ equivariant concordance classes of (PL locally flat) invariant codimension two spheres with the action on the in- 
variant sphere equivalent to $\tau$. (We do not restrict the action $\rho$ on the ambient sphere.) Finally, using the long exact sequence of Proposition 3.2 of [CS2], we may relate $\Gamma_{n}(\Phi)$ and $\Gamma_{n}(F)$ :

$$
\rightarrow L_{n}(Z) \rightarrow \Gamma_{n}(F) \rightarrow \Gamma_{n}(\Phi) \rightarrow L_{n-1}(Z) \rightarrow
$$

placing the relation between two well-known Wall surgery obstruction groups [Br1], [Sh2]. For a more extensive discussion see [CS2, 10.7f]. We also note that the inclusion-transfer sequence of Chapter III (3.13) can be interpreted as

$$
\Gamma_{2 n}(Z \rightarrow e) \rightarrow \Gamma_{2 n}(Z \rightarrow Z / m Z) \rightarrow \Gamma_{2 n}(Z \rightarrow e)
$$

We now proceed to give an algebraic interpretation of Proposition 5.4, by relating $\Gamma_{2 k+2}(F)$ and $E^{\varepsilon}(m ; Z)$. First recall the algebraic definition of $\Gamma_{2 n}\left(\Lambda_{1} \rightarrow \Lambda_{2}\right)$, where $F$ is a ring homomorphism of rings with involution. $\Gamma_{2 n}(F)$ consists of triples $(M, \lambda, \mu)$, where $M$ is a finitely generated $\Lambda_{1}$ module, $\lambda$ is a bilinear form on $M$ with values in $\Lambda_{1}$ and $\mu$ is the associated "self-intersection" form satisfying (i)-(v) of Wall [W2, Chapter V], and such that, after tensoring with $\Lambda_{2}$, the triple is free and unimodular over $\Lambda_{2}$, i.e. in $L_{2 n}\left(\Lambda_{2}\right)$. Null forms are defined to be those for which there exists a submodule $N$ of $M$ on which $\lambda$ and $\mu$ vanish and which, upon tensoring with $\Lambda_{2}$, yield a subkernel in $L_{2 n}\left(\Lambda_{2}\right)$; see [CS2] for details. Now, in our particular case, $\Gamma_{2 n}(Z \rightarrow Z / m Z)$, it is easily shown that $\lambda$ determines the form $\mu$ except for the coefficient of the identity when $n$ is odd (the Kervaire Arf invariant case). Therefore we need only determine $\lambda$ and the Kervaire Arf invariant to identify an element in $\Gamma_{2 n}(F)$.

First, we show how to generalize the results of Wall [W3] to forms of both symmetry over $\Lambda=Z\left[t, t^{-1}\right]$, the ring of Laurent polynomials. Let $V$ be a free $\Lambda$-module and $\phi: V \times V \rightarrow \Lambda$ be an $\varepsilon$-symmetric bilinear form which is nondegenerate, i.e. Ad $\phi: V \rightarrow \operatorname{Hom}_{\Lambda}(V, \Lambda)$ is injective. Let $S \subset \Lambda$ be the $\varepsilon$-symmetric elements under the standard involution ${ }^{-}$on $\Lambda$ (i.e. $\bar{x}=\varepsilon x$ ) and $E$ in $S$, the even elements (i.e. $2 \mid a_{0}$, where $a_{0}$ is the coefficient of the identity). Note that $E=S$ if $\varepsilon=-1$, and that $E$ is an ideal in $S$ (for $s$ in $S$ can be written as $s=\Sigma a_{i}\left(t^{i}+t^{-i}\right)$ so that

$$
s \cdot e=\left(a_{0}+\sum_{i>0} a_{i}\left(t^{i}+t^{-i}\right)\right)\left(b_{0}+\sum_{i>0} b_{i}\left(t^{i}+t^{-i}\right)\right)
$$

has coefficient of $t^{0}=a_{0} b_{0}+\sum_{i>0} 2 \varepsilon a_{i} b_{i}$, which is even since $e$ is in $E$, hence $s \cdot e$ is in $E$ ). Assume also that $\phi$ is an even form, i.e. $\phi(x, x)$ is in $E$ for all $x$ in $V$. Since $\Lambda$ is an integral domain, we may define $F=$ quotient field of $S$ in $Q(t)$. Consider $V^{\#}=\left\{y\right.$ in $V \otimes Q(t): \phi^{\#}(x, y)$ is in $\Lambda$ for all $x$ in $\left.V\right\}$, where $\phi^{\#}$ is the obvious extension of $\phi$ in $V \otimes Q(t)$. On $\bar{V}=V^{\#} / V$, there is a quotient bilinear form $\bar{\phi}: \bar{V} \times \bar{V} \rightarrow Q(t) / \Lambda$ and $\bar{q}: \bar{V} \rightarrow F / E$, an associated 
quadratic form. In this situation we now prove the following theorem (generalizing Wall [W3] to the ring $\Lambda$ and the skew symmetric case) which states that $(\bar{V}, \bar{\phi})$ determines $(V, \phi)$ up to stable equivalence under direct sum with a unimodular even form.

Proposition 5.5 (Compare Wall [W2]). Let $(V, \phi)$ and $(W, \lambda)$ be nondegenerate even e-symmetric bilinear forms over $\Lambda=Z\left[t, t^{-1}\right]$ and let $f:\left(\bar{V}, \bar{\phi}, \bar{q}_{1}\right)$ $\rightarrow\left(\bar{W}, \bar{\lambda}, \bar{q}_{2}\right)$ be an isometric isomorphism over quadratic forms. Then there exist homomorphisms $h: V^{\#} \rightarrow V$ and $g: V^{\#} \rightarrow W^{\#}$ such that:

(i) $i=(h, \mathrm{Id}, g): V^{\#} \rightarrow V+V^{\#}+W^{\#}=H(V)+W^{\#}$ is an isometric embedding (with $H(V)$ given the standard hyperbolic form derived from $\phi$ ).

(ii) $H(V)+W^{\#} \cong M+i\left(V^{\#}\right)$, where $M$ is the orthogonal complement of $i\left(V^{\#}\right)$.

(iii) The induced form on $M$ is even and unimodular.

Proof (Adapted from Wall [W3]). Since $V$, hence $V^{\#}$, is free over $\Lambda$ we may define $g$ on a free basis $x_{i}$ for $V^{\#}$ and extend $\Lambda$-linearly. Set $g\left(x_{i}\right)=z_{i}$ in $W^{\#}$ where $z_{i}$ satisfies $f\left(p_{1}\left(x_{i}\right)\right)=p_{2}\left(z_{i}\right)$, where $p_{1}$ is the projection $p_{1}: V^{\#} \rightarrow$ $V^{\#} / V$ and $p_{2}: W^{\#} \rightarrow W^{\#} / W$. Now the equation $\gamma(x, y)=\phi(x, y)-$ $\lambda(g(x), g(y))$ defines an $\varepsilon$-symmetric bilinear form on $V^{\#}$, since $g$ induces $f$, which is an isomorphism of the associated quadratic forms, $\gamma$ is even and takes values in $\Lambda$. Again making choices, we can find a bilinear form $\mu$ : $V^{\#} \times V^{\#} \rightarrow \Lambda$ satisfying $\gamma(x, y)=\mu(x, y)+\mu(y, x)$. Finally we define $h$ : $V^{\#} \rightarrow V$ by $\phi(x, h(y))=\mu(x, y)$ using the duality of $V$ and $V^{\#}$ under $\phi$. $i=(h, \mathrm{Id}, g)$ is an isometry since

$$
\phi(x, y)-\lambda(g(x), g(y))=\gamma(x, y)=\phi(x, h(y))+\phi(y, h(x)) .
$$

Now $\phi$ is nondegenerate so $M \cap i\left(V^{\#}\right)=0$. For $v$ in $V$, define $V^{\#} \rightarrow V$ by $u \mapsto \phi(-v, h(u))+\lambda(g(-v), g(u))$ which maps into $\Lambda$ so, by duality, is given by $u \mapsto-\phi(d(v), u)$ for some element $d(v)$ in $V$. Now

$$
\begin{aligned}
\phi(h(v)+d(v), u) & =\phi(h(v), u)-\phi(-v, h(u))-\lambda(g(-v), g(u)) \\
& =i(v) \cdot i(u)=\phi(v, u) \text { since } i \text { is an isometry. }
\end{aligned}
$$

Since this is true for all $u$ in $V^{\#}, h(v)+d(v)=v$. By definition of $d(v)$, $(d(v),-v, g(-v))$ is in $M$ and $(h(v), v, g(v))$ is in the image of $i$, so $d(v)+h(v)=v$ is in the sum, i.e. $V \subset M+i\left(V^{\#}\right)$. Now the projection of $M$ onto the last two factors in (i) is onto $V^{\#}+W$ (and, in fact, injective) since, for $v$ in $V^{\#}$ and $w$ in $W, u \mapsto \phi(v, h(u))+\lambda(w, g(u))$ is a linear map $V^{\#} \rightarrow \Lambda$, so given by $u \mapsto-\phi(x, u)$ for some (unique) $x$ in $V$, but then $(x, v, w)$ is in $M$. So $V+V^{\#}+W \subset M+i\left(V^{\#}\right)$, and since $g$ induces $f$ we easily obtain (ii).

Let $\left(u_{i}, v_{i}, w_{i}\right)$ be in $M(i=1,2)$. Under the induced form their inner 
product is

$$
\begin{aligned}
\phi\left(u_{1}, v_{2}\right)+ & \phi\left(u_{2}, v_{1}\right)+\lambda\left(w_{1}, w_{2}\right) \\
= & -\phi\left(\psi_{1}, h\left(v_{2}\right)\right)-\lambda\left(w_{1}, g\left(v_{2}\right)\right) \\
& -\phi\left(v_{2}, h\left(v_{1}\right)\right)-\lambda\left(w_{2}, g\left(v_{1}\right)\right)+\lambda\left(w_{1}, w_{2}\right) \quad \text { by the definition of } M, \\
= & -\gamma\left(v_{1}, v_{2}\right)-\lambda\left(w_{1}, g\left(v_{2}\right)\right)-\lambda\left(w_{2}, g\left(v_{1}\right)\right)+\lambda\left(w_{1}, w_{2}\right) \\
= & \lambda\left(g\left(v_{1}\right), g\left(v_{2}\right)\right)-\phi\left(v_{1}, v_{2}\right)-\lambda\left(w_{1}, g\left(v_{2}\right)\right) \\
& -\lambda\left(w_{2}, g\left(v_{1}\right)\right)+\lambda\left(w_{1}, w_{2}\right) \\
= & \lambda\left(w_{1}-g\left(v_{1}\right), w_{2}-g\left(v_{2}\right)\right)-\phi\left(v_{1}, v_{2}\right),
\end{aligned}
$$

which is independent of the $u_{i}$.

Now, for $(u, v, w)$ in $M, \phi(u, x)+\phi(v, h(x))+\lambda(w, g(x))=0$ for all $x$ in $V^{\#}$. Now the first two terms are in $\Lambda$ so the projection of $w$ in $W^{\#} / W$ is orthogonal to $p_{1}(g(x))$ for all $x$ in $V^{\#}$. But the form on $W$, hence $W^{\#} / W$, is nondegenerate so $w$ must be in $W$, hence $w_{1}$ and $w_{2}$ in (*) must be in $W$. So reducing (*) modulo $V$ and $W$, we see that the induced form on $M$ is even and takes values in $\Lambda$, since $g$ induces $f$ which is an isomorphism of associated quadratic forms.

Let $s: M \rightarrow \Lambda$ be a homomorphism. By the previous analysis of $M$ (the projection $M \rightarrow V^{\#}+W$ is an isomorphism), we may define $\tau_{1}: V+W \rightarrow$ $M \rightarrow^{s} \Lambda$. Now, $\left(\begin{array}{l}\text { Id } \\ 0\end{array} \pm G\right)$, where $G$ is a matrix representative for $g$, defines an automorphism of $V+W$ (and also of $V^{\#}+W^{\#}$ ). So, by duality, there is a $v_{0}$ in $V^{\#}, w_{0}$ in $W^{\#}$ so that

$$
t(v, w+g(v))=-\phi\left(v_{0}, v\right)+\lambda\left(w_{0}-g\left(v_{0}\right), w\right) .
$$

Furthermore, this equation holds also for $v$ in $V^{\#}$ and $w$ in $W$, hence, on $M$ we have

$$
s\left(u, v, w^{\prime}+g(v)\right)=-\phi\left(v_{0}, v\right)+\lambda\left(w_{0}-g\left(v_{0}\right), w^{\prime}\right)
$$

or

$$
s(u, v, w)=-\phi\left(v_{0}, v\right)+\lambda\left(w_{0}-g\left(v_{0}\right), w-g(v)\right) .
$$

Now, if we can demonstrate that $w_{0}$ is in $W$, then there is a $u_{0}$ so that $\left(u_{0}, v_{0}, w_{0}\right)$ is in $M$, and by (*) $s$ is given by pairing with $\left(u_{0}, v_{0}, w_{0}\right)$, proving unimoduality.

Now $s(u, v, w)$ takes values in $\Lambda$ on $M$, so projecting:

$$
\begin{aligned}
0 & \equiv-v_{0} \cdot v+\left(w_{0}-g\left(v_{0}\right)\right) \cdot(w-g(v)) \\
& \equiv-v_{0} \cdot v+\left(w_{0}-g\left(v_{0}\right)\right) \cdot(-g(v)) \text { since }(u, v, w) \text { in } M \text { implies } w \text { in } W \\
& \equiv w_{0} \cdot g(-v) \quad \text { since } g \text { projects to an isometry, }
\end{aligned}
$$

but this holds for all $v$ in $V^{\#}$ so $w_{0}$ is in $W$. 
Let $(W, f)$ be an element of $S_{F}\left(D^{2 k} \times S^{1}\right)$, i.e. $f:(W, \partial W) \rightarrow\left(D^{2 k} \times S^{1}\right.$, $\left.S^{2 k-1} \times S^{1}\right)$ is a homology equivalence restricting to a homotopy equivalence on the boundary. By the fact $\pi_{1}(G / 0)=0$, there is a normal cobordism $\left(M^{2 k+2}, \partial M\right)$ from $W$ to $D^{2 k} \times S^{1}$. Since $f$ is a homology equivalence, there is a surjective map $\pi_{1} M \rightarrow Z$. Let $\tilde{M}$ be the associated cover. By standard techniques, one can do surgery, first in low dimensions, then in dimension $(k+1)$, on the interior of $M$, to make the intersection form $\lambda$ on $W=$ $H_{k+1}(\tilde{M} ; Z)$ nondegenerate over $\Lambda$ (or rank two with a nonstandard hyperbolic plane, i.e. the Kervaire Arf invariant case which we are neglecting). We then have the following computation:

THEOREM 5.6. Let $\left(J^{2 k+1}, K ; T\right)$ be an invariant knot. The $\lambda$ form associated as above to $\theta(K / T)$ (see [CS2, 10.3]) (the structure obtained from the complement of $K / T$ in the orbit space) is, up to stable equivalence, given by the $\varepsilon$-symmetric $\left(\varepsilon=(-1)^{k+1}\right) \Lambda$-bilinear form associated to

$$
[(\Delta(t)-1) /(t-1)]\{(t-1) D+\operatorname{Id}\} Q,
$$

where $\Delta(t)$ is the normalized $(\bar{\Delta}=\Delta)$ Alexander polynomial of $s_{1}, D$ is a matrix associated to the isometry $s_{1}$, and $Q$ is the associated intersection matrix on a free $\Lambda$-module of the appropriate rank.

Remarks. (1) The above form (on $W^{\#} / W$ ) is due to H. F. Trotter [T] for the ordinary knot concordance case.

(2) Equivalence classes of elements of $\Gamma_{2 n}\left(\Lambda_{1} \rightarrow \Lambda_{2}\right)$ under stable equivalence by forms unimodular over $\Lambda_{1}$ are in 1-1 correspondence with $\Gamma_{2 n}\left(\Lambda_{1} \rightarrow\right.$ $\left.\Lambda_{2}\right)$ /image $L_{2 n}\left(\Lambda_{1}\right)$. Note also that, up to stable equivalence, the form $\lambda$ (together with an associated $\mu$ form) determines an element in $\Gamma_{2 n}(F)$ depending only on the equivariant algebraic concordance class of $\left(Q ; s_{0}, s_{1}\right)$.

(3) One may easily verify that $\Delta(\zeta)$ is a unit in $Z$ [ $\zeta], \zeta$ a primitive $m$ th root of unity iff $W^{\#} / W \otimes Z[Z / m Z]=0$, i.e. $\lambda \otimes Z[Z / m Z]$ is unimodular.

(4) Theorem 5.6 gives the theoretical possibility of calculating the multisignature and, hence, determining the structure on $J / T$ from that of $K / T$ and invariants of $X / T$ and the normal bundle (see Chapter IV).

(5) This theorem is the desired algebraic interpretation of Proposition 5.4.

Proof. Let $M$ be a normal cobordism to $D^{2 k} \times S^{1}$ for $\theta(K / T)$. Without loss of generality we may assume $M$ is $k$-connected (except for $\pi_{1} K=Z$ ). By the (singular) homology exact sequence for $(\tilde{M}, \partial \tilde{M}), \tilde{M}=$ universal cover of $M$, we have the following exact sequence of $\Lambda$-modules:

$$
0 \rightarrow H_{k+1}(\partial \tilde{M}) \rightarrow H_{k+1}(\tilde{M} ; Z) \rightarrow H_{k+1}(\tilde{M}, \partial \tilde{M}) \rightarrow H_{k}(\partial \tilde{M}) \rightarrow 0 .
$$

But, by the theorem of Milnor [M3, in particular, Remark 1, p. 126] on the duality properties of infinite cyclic coverings, $H_{\mathrm{k}+1}(\partial \tilde{M})=0$. So we are reduced to the situation 


$$
0 \rightarrow W \rightarrow W^{\#} \rightarrow W^{\#} / W \rightarrow 0 .
$$

where $W=H_{k+1}(\tilde{M} ; Z)$ and the form on $W^{\#}$ is the usual cup product pairing on the Lefschetz dual module $H^{k+1}(\tilde{M} ; Z)$ with values in $\Lambda$.

We now identify the induced form on $W^{\#} / W=H_{k}(\partial \tilde{M})=H_{k}(\tilde{X} / T)$, where $X$ is the complement of an invariant tube about $K$ in $J$. In fact, it will be more convenient to consider the Poincare dual module $H^{k+1}(Y)$ with $Y=\tilde{X} / T$. Since this module is $\Lambda$-torsion, the Bockstein associated to $0 \rightarrow \Lambda$ $\rightarrow F=Q(t) \rightarrow F / \Lambda \rightarrow 0$ is an isomorphism, $\delta^{*}: H^{k}(Y ; F / \Lambda) \rightarrow$ $H^{k+1}(Y ; \Lambda)$. The coefficient pairing $F / \Lambda \rightarrow \Lambda \rightarrow F / \Lambda$ induces

$$
H^{k}(Y ; F / \Lambda) \times H^{k+1}(Y ; \Lambda) \rightarrow H^{2 k+1}(Y ; F / \Lambda)=F / \Lambda .
$$

So we obtain an $F / \Lambda$-valued form on $H^{k+1}(Y ; \Lambda)$ by $u \cdot v=u \cup\left(\delta^{*}\right)^{-1}(v)$, which is easily shown to be the induced form on $W^{\#} / W$ in (*) above.

H. F. Trotter [T, p. 4 and 1.7], who studied the induced form on $W^{\#} / W$ in this context, has shown that the $\varepsilon$-symmetric matrix as in the statement of the theorem induces the correct form on the boundary. As the form on the boundary is natural (hence isomorphic forms are induced by different choices on $M$ ), any choice for $M$ must induce a stably equivalent form by Proposition 5.5.

The methods of this paper can be extended to give some information on the problem of doing surgery on the complement up to homotopy equivalence using the work of Levine [L5] and Trotter [T]. We will only state the results without proof.

Definition 5.7. Let $M^{m}, N^{n}$ be smooth compact manifolds and $i: M \rightarrow N$ a fixed proper embedding (i.e. $i^{-1}(\partial N)=\partial M$ ). An $h$-concordance of $i$ is a proper embedding $F: M \times I \rightarrow N \times I$ such that $F_{0}=i, F_{1}$ is proper on $N \times 1,\left.F\right|_{\partial M \times I}=\left.i\right|_{\partial M} \times \operatorname{Id}_{I}$ and $N \times I-F(M \times I)$ is a relative $h$-cobordism.

Let $F: \Lambda_{1} \rightarrow \Lambda_{2}$ be a ring homomorphism of rings with involution and $(M, \lambda, \mu)$ as in the definition of the $\Gamma$-groups [CS2]. Let $\varrho_{2 n}(F)$ be the monoid of equivalence classes of isomorphism classes of forms $(M, \lambda, \mu)$ under stable equivalence by direct sum with forms unimodular over $\Lambda_{1}$.

TheOREM $5.8(k>2)$. Let $\left(J^{2 k+1}, K ; T\right)$ be a simple invariant knot. The induced embedding on the orbit space is $h$-concordant to an embedding with a trivial knot complement iff an obstruction $\beta(J, K ; T)$ in $\varrho_{2 n}(Z \rightarrow Z / m Z)$ is zero and all obstructions are realizable.

For the relationship between $h$-concordance and isotopy in codimension 2 see Hatcher [H]. 


\section{BIBLIOGRAPHY}

[B1] H. Bass, The Dirichlet unit theorem, induced characters and Whitehead groups of finite groups, Topology 4 (1965), 391-410.

[B2] __, $K_{2}$ des corps globaux, Seminaire Bourbaki, $23^{e}$ annee, 1970/71, no. 394.

[Bla] R. C. Blanchfield, Intersection theory of manifolds with operators with applications to knot theory, Ann. of Math. (2) 65 (1957), 340-356.

[Br1] W. Browder, Manifolds with $\pi_{1}=Z$, Bull. Amer. Math. Soc. 72 (1966), 234-244.

[Br2] - Surgery and the theory of differentiable transformation groups, Proc. Tulane Symposium on Transformation Groups, Springer, Berlin, 1968, pp. 1-46.

[Br3] _ Free $Z_{p}$-actions on homotopy spheres, Topology of Manifolds, Markham, Chicago, Ill., 1970, pp. 217-226.

[Br4] __ Surgery on simply-connected manifolds, Springer, New York, 1972.

[BL] W. Browder and G. R. Livesay, Fixed point free involutions on homotopy spheres, Tôhoku Math. J. 25 (1973), 69-88.

[BPW] W. Browder, T. Petrie and C. T. C. Wall, The classification of free actions of cyclic groups of odd order on homotopy spheres, Bull. Amer. Math. Soc. 77 (1971), 455-459.

[Bu] E. Burger, Über gruppen mit verschlingungen, J. Reine Angew. Math. 188 (1950), 193-200.

[CS1] S. Cappell and J. Shaneson, Submanifolds, group actions and knots. I, II, Bull. Amer. Math. Soc. 78 (1972), 1045-1052.

[CS2] __ The codimension two placement problem and homology equivalent manifolds, Ann. of Math. (2) 99 (1974), 277-348.

[D] A. Dold, Lectures on algebraic topology, Springer, Berlin, 1972.

[FM] R. H. Fox and J. Milnor, Singularities of two spheres in four space and cobordism of knots, Osaka J. Math. 3 (1966), 257-267.

[F1] R. H. Fox, Free differential calculus. III: Subgroups, Ann. of Math. (2) 64 (1956), $407-419$. [F2] , On knots which are fixed under a periodic transformation of the 3-sphere, Osaka J.

Math. 10 (1958), 31-35.

[F3] _ A quick trip through knot theory, Topology of Three Manifolds and Related

Topics (M. K. Fort, Jr., editor), Prentice-Hall, Englewood Cliffs, N. J., 1962.

[Fu] F. B. Fuller, A relation between degree and linking numbers, Algebraic Geometry and Topology, Princeton Univ. Press, Princeton, N. J., 1957, pp. 258-262.

[G] C. McA. Gordon, Knots whose branched cyclic coverings have periodic homology, Trans. Amer. Math. Soc. 168 (1972), 357-370.

[H] A. Hatcher, Concordance and isotopy of smooth embeddings in low codimensions, Invent. Math. 21 (1973), 223-232.

[Hi] F. E. P. Hirzebruch, Singularities and exotic spheres, Seminaire Bourbaki, $19^{\circ}$ annee, $1966 / 67$, no. 314.

[KaMa] M. Kato and Y. Matsumoto, Simply connected surgery of submanifolds in codimension two, J. Math. Soc. Japan 24 (1972), 586-608.

[K1] M. Kervaire, Les noeuds de dimension superieure, Bull. Soc. Math. France 93 (1965), 225-271.

[K2] __ Knot cobordism in codimension two, Lecture Notes in Math., vol. 197, Springer, Berlin, 1971, pp. 83-105.

[KM] M. Kervaire and J. Milnor, Groups of homotopy spheres. I, Ann. of Math. (2) 77 (1963), 504-537.

[KV] M. Kervaire and A. Vasquez, Simple connectivity and the Browder-Novikov theorem, Trans. Amer. Math. Soc. 126 (1967), 508-513.

[La] S. Lang, Algebra, Addison-Wesley, Reading, Mass., 1965.

[Le? S. Lefschetz, Algebraic topology, Amer. Math. Soc. Colloq. Publ., vol. 27, Amer. Math. Soc., Providence, R. I., 1942.

[L1] J. Levine, Unknotting spheres in codimension two, Topology 4 (1965), 9-16.

[L2] _ Polynomial invariants of knots in codimesion two, Ann. of Math. (2) 84 (1966), 537-554. 
[L3] __ Knot cobordism in codimension two, Comment. Math. Helv. 44 (1968), 229-244.

[LA] __ Invariants of knot cobordism, Invent. Math. 8 (1969), 98-110.

[L5] An algebraic classification of some knots of codimension two, Comment. Math. Helv. 45 (1970), 185-198.

[LdM1] S. Lopez de Medrano, Involutions on manifolds, Springer, New York, 1971.

[LdM2] _ , Nudos invariantes bajo involuciones. I, An. Inst. Mat. Univ. Nac. Autónoma de México 8 (1969), 81-90.

[LdM3] __ Invariant knots and surgery in codimension two, Actes Congres Internat. Math. 2, Gauthier-Villars, Paris, 1970.

[Ma] Y. Matsumoto, Knot cobordism groups and surgery in codimension two, J. Fac. Sci. Univ. Tokyo Sect. IA Math. 20 (1973), 253-317.

[M1] J. Milnor, A duality theorem for Reidemeister torsion, Ann. of Math. (2) 76 (1962), 137-147.

[M2] _ _ Whitehead torsion, Bull. Amer. Math. Soc. 72 (1966), 358-426.

[M3] _ Infinite cyclic coverings, Conference on the Topology of Manifolds (J. G.

Hocking, editor), Prindle, Weber and Schmidt, Boston, Mass. 1968, pp. 115-133.

[M4] __, On isometries of inner product spaces, Invent. Math. 8 (1969), 83-97.

[M5] _ Introduction to algebraic K-theory, Ann. of Math. Studies, No. 72, Princeton Univ. Press, Princeton, N. J., 1971.

[M6] Lectures on the h-cobordism theorem, Notes by L. Siebenmann and J. Sondow, Princeton Univ. Press, Princeton, N. J., 1965.

[Rei] K. Reidemeister, Durschnitt und schnitt von Homotopieketten, Monatsh. Math. Phys. 48 (1939), 226-239.

[Ro] R. A. Robertello, An invariant of knot cobordism, Comm. Pure Appl. Math. 18 (1965), 543-555.

[Sch] R. E. Schultz, Smooth structures on $S^{p} \times S^{q}$, Ann. of Math. (2) 90 (1969), 187-198.

[Sei] H. Seifert, Uber das Geschlecht von Knoten, Math. Ann. 110 (1934), 571-592.

[St] N. Stoltzfus, Unraveling the integral knot concordance group, Mem. Amer. Math. Soc., No. 192 (1977).

[Sh1] J. Shaneson, Embeddings with codimension two of spheres in spheres and h-cobordisms of $S^{1} \times S^{3}$, Bull. Amer. Math. Soc. 74 (1968), 972-974.

[Sh2] — Wall's surgery obstruction group for $Z \times G$, Ann. of Math. (2) 90 (1969), 296-334.

[Sp] E. H. Spanier, Algebraic topology, McGraw-Hill, New York, 1966.

[T] H. F. Trotter, On S-equivalence of Seifert matrices, Invent. Math. 20 (1973), 173-207.

[W1] C. T. C. Wall, Diffeomorphisms of four-manifolds, Proc. London Math. Soc. 39 (1964), 131-140.

[W2] __ Surgery on compact manifolds, Academic Press, New York, 1970.

[W3] __, Quadratic forms on finite groups. II, Bull. London Math. Soc. 4 (1972), 156-160.

Department of Mathematics, louisiana State University, Baton Rouge, louisiana 70803 